\title{
Pharmacoscintigraphic evaluation and antidiabetic efficacy of gliclazide-loaded 99mTc-labelled mucoadhesive microspheres
}

\author{
Ripunjoy Bordoloi ${ }^{1,2^{*}} \mathbb{D}$, Abdul Baquee Ahmed ${ }^{3}$ and Kunal Bhattacharya ${ }^{2}$
}

\begin{abstract}
Background: The current study was carried out to evaluate the possible application of Musa balbisiana starch in formulation of mucoadhesive microsphere for oral delivery of gliclazide (GLZ). The study objective was to improve the oral bioavailability along with prolongation of its duration of action for a better glycaemic control. Ionic gelation technique was employed in formulating the dosage form. Optimization of the batches was carried out by response surface methodology using $3^{2}$ full factorial designs. The microsphere prepared was characterized for several parameters along with its in vitro release study. The gastrointestinal transit of the optimized batch of prepared microspheres after oral administration was studied in rabbits by using the gamma scintigraphy technique utilizing $99 \mathrm{mTc}$ as the labelling agent in the presence of stannous chloride. Also, the optimized batch was studied for its pharmacokinetic parameters. Moreover, the antidiabetic efficacy of the prepared microsphere was evaluated in rats by using the streptozotocin (STZ)-induced diabetic model.

Results: The factorial design experiment resulted in an optimum formulation coded as F8. The compatible nature of the drug and excipient was revealed from FTIR, DSC and IST studies. The scanning electron micrographs also showed the occurrence of spherical microspheres having a smooth surface. The in vitro release study provided an evidence of an initial burst effect that was followed by a prolong release phase. The pharmacokinetic parameters justified the ability of the prepared dosage form in sustaining the drug release with a 2.7-fold enhancement in drug bioavailability. The images obtained during the gamma scintigraphy study suggested the gastro-retentive nature of the dosage form with the gastro-retentive ability for more than $4 \mathrm{~h}$. Also, the pharmacodynamics study carried out in diabetic rat model confirmed about the better efficacy of the dosage form in lowering the elevated blood glucose level.

Conclusion: The overall study data provide valuable information about the potential of this banana starch in formulation of a mucoadhesive dosage form that can be used for enhancement of bioavailability of drug-like gliclazide which in turn can provide a beneficial effect in the management of diabetes.
\end{abstract}

Keywords: Gliclazide, Mucoadhesive microspheres, Gamma scintigraphy, 99mTc-pertechnetate, Ionic gelation

\section{Background}

There is always a major challenge for developing an orally active sustained release dosage form. The challenge lies not only in sustaining the pattern of release

\footnotetext{
*Correspondence: ripun.bordoloi@gmail.com

${ }^{1}$ Assam Science and Technology University (ASTU), Tetelia Road, Jalukbari,

Assam 781013, India

Full list of author information is available at the end of the article
}

from the formulation but also delaying the gastric emptying in account of which the dosage form will be retained mostly in the gastro-intestinal (GI) tract till all the active medicament is completely released within the time specified [1]. The absorption pattern of drugs and medicaments from the GI tract is indeed a complex process which is influenced by several variables and is basically related to the time of contact of the dosage form with the mucosal lining of small intestines. There are already 
several reported dosage forms which are specifically designed in view of gastro-retention, one such approach is mucoadhesive microspheres. Microspheres, which are sometimes defined as micro-particles, are small spherical particles with its size ranging from 1 to $1000 \mu \mathrm{m}$. The microspheres can be made up of either natural or synthetic polymers [2]. The shorter residence time of this delivery system, specifically at the absorption site, is the main limitation, which decreases the utility. This major limitation can only be overcome if an approach for establishing a firm contact of the drug delivery system with the absorbing membrane is carried out. This can be achieved by incorporating bioadhesion characteristics to the micro-particles, thereby developing delivery systems referred to as "bioadhesive micro particles" [3]. The concept of bioadhesion also combines the advantage such as enhancement of drug continuance mostly at the absorption site, and it has been applied to various formulations to target different parts in the GI tract.

The concept of bioadhesion also known as mucoadhesion has been of a greater interest in development of controlled drug delivery system for improving the buccal, nasal and oral administration of drugs. These bioadhesive polymers usually contain a hydroxyl or some carboxyl groups that potentiate the formation of hydrogen bonds with the mucosal surfaces. There are several polymers that have been investigated as bioadhesive drug carriers which include poly-acrylic acid (PAA), poly-methacrylic acid, cellulose derivatives, polyethylene oxide, lechitin and chitosan. Although the PAA and its available crosslinked commercial forms, Carbopol ${ }^{\circledR}$ and Poly carbophil $^{\circledR}$, exhibit strong bioadhesive properties, they were found to have tremendous mucosal irritation thereby limiting their use as bioadhesive drug carriers [4]. Hence, there is always an attempt to develop natural polymers as bioadhesive drug delivery systems.

Utilizing naturally available polymers in formulating a dosage form is of greater interest owing to its ease of availability and better tolerability [3]. Natural polymers can be obtained from both the plant as well as animal kingdom [5]. These are mostly occurring as high molecular weight compounds comprising of monosaccharide units joined together by glycoside linkage [6]. Amongst the various naturally occurring polymers, alginates are most widely used. These alginates polysaccharides contain two monomeric units, namely $\beta$-D-mannuronic acid (M) and $\alpha$-L-guluronic acid (G) [7]. Species of marine brown algae like Laminaria hyperborean, Ascophullum nodosum, Macrocystis pyrifera, etc., are primarily the source from which alginates can be obtained [8]. An essential feature of this polysaccharide is that it tends to undergo ionotropic gelation readily in a solution containing water. This process is accelerated with various positively charged ions like $\mathrm{Ca}^{2+}, \mathrm{Ba}^{2+}, \mathrm{Pb}^{2+}$, $\mathrm{Cu}^{2+}, \mathrm{Cd}^{2+}, \mathrm{Zn}^{2+}$ that are mostly divalent and in the presence of $\mathrm{Al}^{3+}$ that are mostly trivalent. This technique in an aqueous medium (water) mostly preponderates because of the ionic interaction and the intermolecular bonding between the $\mathrm{COOH}$ groups residing on the polymer backbone with the cations. Even though this polymer has mucoadhesive properties, still the primary problem is that the cross-linked alginate beads are fragile [9]. An approach is hence necessary to blend different mucoadhesive polymers along with alginates [10]. Starch is widely used as a mucoadhesive polymer, and it is often considered the second most renewable polymer that is cost-effective, safe, and fully biodegradable [11]. Commercially, it can be collected from several familiar sources that include maize (Zea mays L.), wheat (Triticum vulgare L.), rice (Oryza sativa L.), cassava (Manihot esculenta Crantz), plant tubers, e.g. potato (Solanum tuberosum L.). [12] The property of starch can vary depending on the source of origin and the method employed for processing it.

The starch obtained from various sources of banana has received tremendous attention in relation to its structural and physiochemical properties [13] Naturally occurring starch is a good texture stabilizer and a regulator in food systems, but it has limitations like low shear resistance, thermal resistance, thermal degradation and also possess high tendency towards retro-gradation that limits its use in some industrial food applications [14]. The modification of starch involves altering its physical and chemical characteristics in order to improve its functional properties that can be used to tailor starch for specific food applications. The modification is mostly achieved through derivatization such as etherification, esterification, cross-linking grafting of starch decomposition (acid or enzymatic hydrolysis and oxidation of starch) or physical treatment of starch using heat or moisture. The chemical modification includes the addition of functional groups to the starch molecules which results in some marked altered physiochemical properties. Such modifications of natural granular starches profoundly alter its gelatinization, pasting and retro-gradation behaviour. Starch used in the current study was obtained from Musa balbisiana species that was followed with some physical and chemical modification. Musa balbisiana also commonly known as "Bhim kol" or "Athia kol" is a common species of banana found in Assam and North East India. The use of this species of banana is restricted to only food because of its higher nutritive value. Although starch can be easily gelatinized or dissolved in water, it is still difficult to process it owing to its high viscosity and thickening properties occurring at low solid content. The utilization of starch as a pharmaceutical excipient 
requires some specific properties that are not usually present in the native starch. There is requirement of application of numerous physical and chemical modifications in the native starch for imparting properties that are useful for particular applications. Acetylation is the esterification of starch polymers where the hydroxyl groups of the $\alpha$-D-glucopyranose unit are converted to acetyl groups to form starch acetates. Imparting acetylation results in alteration of a wide range of functional characteristics of native starches such as conferring higher peak viscosity (PV) and paste clarity and increasing freeze thaw stability. Moreover, starch acetate shows stability and resistance to retro-degradation and impart thickening in diverse foods.

The drug used in the present study is gliclazide classified under BCS class II which is an anti-diabetic drug mostly used in management of Type II Diabetes. As per the chemical structure, this drug is categorized as the first-generation sulfonylurea because of a proton releasing sulphonamide group and an aromatic group. However, on the other hand, based on its pharmacological activity, this drug is categorized as a second-generation sulfonylurea that has a high potency and a shorter halflife [15]. A single oral dose of gliclazide resulted in $T_{\max }$ ranging from 2 to $8 \mathrm{~h}$ due to its $\mathrm{pH}-$ dependent and low solubility. Gliclazide has a low volume of distribution with a strong plasma protein binding of about $94.2 \%$, and it undergoes extensive hepatic metabolic biotransformation to produce several inactive metabolites with no circulating active metabolites. In relating with the previously available data, good general tolerability of this drug among the individuals can be predicted. Moreover, another prediction of the minimum rate of secondary failure from this drug molecule can be made based on available study data. The drug is taken mostly by the oral route, and there are several reports revealing in slow and variable absorption pattern among the individuals leading to alteration in its bioavailability. This variation in absorption of gliclazide could be related to its early dissolution in the stomach leading to its more variability in the intestinal absorption. In order to control this, an approach of incorporation of gliclazide in gastro-retentive formulations like mucoadhesive microspheres can be carried out. However, there are no available data in utilization of starch isolated from Musa balbisiana species in formulation of mucoadhesive microspheres loaded with this anti-diabetic drug (Additional file 1: Arrive Checklist).

The current study was carried out to evaluate the possible application MSB starch in the preparation of mucoadhesive microsphere as this starch is having a tremendous potential in imparting mucoadhesive properties and can be a better candidate as a mucoadhesive polymer over the other. MSB starch microsphere loaded with gliclazide was prepared by ionic gelation technique using calcium chloride as the cross-linking agent. The prepared microspheres were characterized in terms of particle size and morphology, percentage yield, drug entrapment efficiency, mucoadhesive study and in vitro drug release study. Furthermore, a $3^{2}$ full factorial design was applied with desirability function in understanding the quality and optimization of gliclazide mucoadhesive microsphere. The factorial design was applied in investigating out the mutual effect of polymer concentration (X1) and concentration of $\mathrm{CaCl}_{2}$ (X2) used as the crosslinking agent on the various responses $(\mathrm{Y})$ like drug loading (\%) indicated as $\mathrm{Y} 1$, bioadhesion (\%) indicated as $\mathrm{Y} 2$ and drug release (\%) after $12 \mathrm{~h}$ of study indicated as $\mathrm{Y} 3$. To relate each response with the factors affecting it, polynomial equations were used. Moreover, response surface plots and contour plots were also drawn and selection of optimum formulation was carried out using the desirability function. The software Design expert that was used provides a reliable information of not only about all the critical values that is necessary to obtain the desired response in the final formulation, but also it provides a valuable information about the possible incompatibilities of the designated independent variables in the formulations on the dependent variable. On the other hand, the present work focused not only on the in vitro characterization but also on the in vivo behaviour of the prepared dosage form. The optimized formulation was subjected to pharmacokinetics evaluation along with assessment of anti-diabetic efficacy using streptozotocin (STZ)-induced diabetes rat model. Furthermore, the assessment of the gastro-retentive behaviour was carried out by using the gamma-scintigraphy technique.

The aim of this work was to develop an effective gliclazide mucoadhesive microsphere by utilizing the starch obtained for Musa balbisiana species for prolonging the release and also improving the oral bioavailability of the drug thereby sustaining its anti-diabetic activity.

\section{Materials \\ Materials}

Gliclazide and Phenytoin were received from Legen Healthcare, India, sodium alginate from Otto Chemie Private Ltd., India, and calcium chloride $\left(\mathrm{CaCl}_{2}\right)$ from Merck Ltd., India. All other chemicals and reagents used for the study purpose were of analytical grade procured from reputed vendors.

\section{Methods}

Isolation, purification and modification of starch

For isolation of starch, unripe fruits of Musa balbisiana species were procured from the local market of 
Guwahati, Assam, India. The fruits were peeled and were cut into $5-6 \mathrm{~cm}$ cubes (500 g total weight) and were immediately rinsed in a solution containing sodium sulphite $(1.22 \mathrm{~g} / \mathrm{L})$ following maceration at a low speed in a grinder for $2 \mathrm{~min}$. The homogenate form was than consecutively shifted through sieve No. 60 \# \& 100 \#, respectively, and washed it with distilled water until the clear filtrate came out. The collected filtrate was further centrifuged for $20 \mathrm{~min}$ at $7000 \mathrm{rpm}$ following which the upper off-white sediments were scrapped out. To the centrifuged mass, water was added and was again subjected to centrifugation for $20 \mathrm{~min}$ at $7000 \mathrm{rpm}$. The entire cycle was repeated for 2-3 times until a clear white starch was obtained. The obtained white starch was washed twice with distilled water before drying in a stove with forced air circulation $3 \mathrm{~h}$ at $45^{\circ} \mathrm{C} \pm 2{ }^{\circ} \mathrm{C}$. It was then stored in polyethylene bags until further use. Physical modifications were attributed by means of pre-gelatinisation, whereas chemical modification was imparted by means of acetylation. About $200 \mathrm{~g}$ of extracted starch was weighed out and was dispersed in $200 \mathrm{ml}$ of distilled water. To the above mixture, mild heating was applied until a paste-like consistency was achieved. This physically modified pre-gelatinized starch was further left to dry at $100{ }^{\circ} \mathrm{C}$ for $24 \mathrm{~h}$ followed by screening through a sieve of mesh size $0.315 \mathrm{~mm}$. The resulting refined and fine powder was finally stored in an air-tight container. An equal amount of extracted starch was again taken for imparting chemical modifications by adding freshly prepared $60 \mathrm{ml}$ of Sodium Hydroxide $50 \%$ and $100 \mathrm{ml}$ of acetic anhydride. On proper mixing, the resultant was dried at $100^{\circ} \mathrm{C}$ for $24 \mathrm{~h}$ following screening through a sieve having mesh size of $0.315 \mathrm{~mm}$ [16-19].

\section{Identification of starch}

The identification of the extracted starch was carried out by taking $1 \mathrm{~g}$ each of natural, pregelatinized and acetylated starch and mixing it with $50 \mathrm{ml}$ of water. The above mixture was than boiled for one minute and cooled. To $1 \mathrm{ml}$ of the above mucilage, iodine solution was added and the resultant colour was observed.

\section{Preparation of gliclazide microsphere by ionic gelation technique}

The microsphere containing GLZ in this study was formulated by utilizing the ionic gelation technique. Initially, sodium alginate and the banana starch polymer were dissolved in $50 \mathrm{ml}$ of deionised water to produce a homogenous polymer solution. The active drug GLZ was added to the polymer solution maintaining a ratio of 1:1 (GLZ: Polymer) and agitated thoroughly to get a homogenous viscous dispersion by using a homogenizer (Remi Motors, India). Finally, the resultant dispersion containing the drug and the polymer was manually added dropwise via a 26-gauge needle to a solution of $\mathrm{CaCl}_{2}$ solution [20].

\section{Optimization of formulation using factorial design}

The optimization of the formulation was carried out by $3^{2}$ full factorial designs. The evaluation of two different factors at three different levels was done. The two factors selected as independent variable here are amount of polymer and cross-linking agent. These two factors are varied, and factor levels were suitably coded as $-1,0$, and +1 , respectively, for low, intermediate and high. The responses like drug loading (\%), bioadhesiveness (\%) and drug release (\%) after $12 \mathrm{~h}$ were considered as dependant variable. In this design approach, evaluation of two factors was carried out at three levels each and performing the experimental trails for all the combinations possible. Any other processing and also the formulation variables were kept constant throughout the entire study. In total, 9 runs (formulations) were designed by using the software Design Expert version 12. (Stat-Ease Minneapolis, MN, USA). Accordingly, the dependent and the independent variable's relationship pattern were studied and the analysis of the same was carried out on gaining the surface response to attain the significant model.

1. Independent levels:

2. Polymer (X1)

3. Cross-linking agent (X2)

4. Dependent levels:

5. Drug Loading (Y1)

6. Bioadhesiveness $(\mathrm{Y} 2)$

7. Drug Release (Y3)

\section{Statistical analysis}

The statistical optimization was performed using Design Expert version 12. (Stat-Ease Minneapolis, MN, USA). All measured data are expressed as mean \pm standard deviation (S.D.). Each of the measurement was done in triplicate $(\mathrm{n}=3)$.

\section{HPLC analysis of Gliclazide}

The chromatographic analysis was carried out on Thermo Scientific (Dionex Ultra 3000plus) HPLC using a stainless steel column $(25 \mathrm{~cm} \times 4 \mathrm{~mm})$ packed with end capped octylsilane bonded to porous silica $(4 \mu \mathrm{m})$ in isocratic elution mode using phosphate buffer $\mathrm{pH} 3.4$ and HPLC grade acetonitrile in the ratio of 20:80 (v/v) as eluent. An aliquot of $20 \mu \mathrm{l}$ sample was injected to the column 
by maintaining the flow rate at $1 \mathrm{ml} / \mathrm{min}$. The eluent was detected at $230 \mathrm{~nm}$. [21]

\section{Drug and excipient compatibility study Fourier transform infrared spectroscopy (FTIR)}

To find out any possible molecular interaction among the drug and the excipients used, the Fourier transform infrared (FTIR) spectroscopy (Bruker) was used. The technique utilizes the potassium bromide $(\mathrm{KBr})$ disc method that involves placing of the disc in the sample holder and scanning it from 4000 to $400 \mathrm{~cm}^{-1}$ with the resolution maintained at $4 \mathrm{~cm}^{-1}$. The analysis was carried out for the pure drug, the polymers used and also the formulation loaded with the drug.

\section{Differential scanning calorimetry (DSC)}

To estimate out the thermal nature of the pure drug, excipient, excipient mixture along with the gliclazideloaded microsphere, the technique of differential scanning calorimetry was used. All the samples were allowed to run in a differential scanning calorimeter (JADE, Perkin Elmer, USA,) at the scanning rate of $10^{\circ} \mathrm{c} / \mathrm{min}$ with the measurements conducted by maintaining the heating range of $40-250{ }^{\circ} \mathrm{C}$ utilizing nitrogen as the purging gas. Furthermore, the evaluations of any possible interactions in the thermograms were carried out using Pyris software (PerkinElmer, Inc., Waltham, MA, USA). [22]

\section{Isothermal stress testing (IST)}

In IST studies, the drug along with different excipients used was weighed directly into a previously cleaned $4 \mathrm{ml}$ glass vials $(\mathrm{n}=2)$ and was subjected to homogenization on a vortex mixture for around 2 min following addition of about $10 \%$ water in each of the vials. The drug-excipient blend was further subjected to mixing with the aid of a glass capillary whose both ends were heat sealed. The capillary was broken in order to prevent any wastage of materials, and finally, each of the vials was sealed using a screw cap lined with Teflon and stored by maintaining a temperature of $50{ }^{\circ} \mathrm{C}$ in a hot air oven. At periodic time interval, the vials containing the samples were visually inspected for any alteration in colour. The analysis of the sample after storing for 3 weeks was carried out by using UV visible spectrophotometer [23]. A vial containing drug excipient mixture stored in a refrigerator without any addition of water to it served as the control sample for the entire analysis. The sample for analysis purpose was prepared by adding $2 \mathrm{ml}$ of methanol to all the vials. The entire mixture was subjected to homogenization and was transferred to a $100 \mathrm{ml}$ volumetric flask. The vials were further rinsed with methanol twice, and the final volume was made up to the mark. Finally, the samples were centrifuged and the superannuated liquid was filtered through a nylon filter and analysis was carried out for estimating the drug content after proper dilution with the same solvent. In addition, the spectral analysis by peak matching technique was also performed which gives an indication about the change in wavelength maxima in case of the pure drug as well as the drug excipient mixture carried out for both the control as well as the stressed sample.

\section{Characterization of prepared microsphere Production yield}

The yield of production for the microsphere prepared was calculated by determining the weight of the final dried microsphere in context to the total amount of the drug and the polymer used for the preparation. Finally, the percentage of production yield was calculated by using the formula mentioned below

$$
\begin{aligned}
& \text { Production Yield } \\
& =\frac{\text { Mass (Microsphere) }}{\text { Theoretical Mass (Polymer }+ \text { Drug) }} \times 100
\end{aligned}
$$

\section{Bulk density}

The estimation of Bulk Density of the prepared microsphere was carried out by accurately weighing out microsphere $(\mathrm{Wm})$ and then transferring it into a graduated cylinder of $100 \mathrm{ml}$ volume in order to attain the apparent volume $(\mathrm{V})$ in between 50 and $100 \mathrm{ml}$. The following formula was used in estimating out bulk density in gram/ millilitre

$$
\text { Bulk density }(\tilde{n} 0)=\frac{M}{V_{\mathrm{o}}}
$$

where $M=$ powder mass, $V_{\mathrm{o}}=$ powder volume.

\section{Angle of repose}

This is a common technique for estimation of the flow property of the prepared microsphere which was carried out by using the fixed funnel method. In this current method, a funnel is used and is fixed to a stand in a way such that its tip achieves a height of $6 \mathrm{~cm}$ when measured from the surface. A sufficient amount of microsphere was then allowed to pass through the funnel so that if forms a pile. Finally, the angle of repose was estimated by measuring out the radius and height of the heap of the pile and putting the values in the formula given below.

$$
\operatorname{Tan} \theta=\frac{H}{R}
$$

Here, $H=$ height of the pile from the surface, $R=$ radius of the pile. 


\section{Drug loading and entrapment efficiency}

Microsphere equivalent to $100 \mathrm{mg}$ was weighed out accurately and transferred to a mortar and pestle and crushed. To the above crushed powdered microsphere, $500 \mathrm{ml}$ of phosphate buffer solution (PBS) having $\mathrm{pH}$ of 7.4 was added and was kept aside for $48 \mathrm{~h}$ with occasional shaking. The temperature condition for this study was maintained at $37 \pm 0.5^{\circ} \mathrm{C}$. After the specified time, the mixture was filtered to remove the polymer debris generated as a result of disintegration of the microcapsules. The estimation of the drug content was carried out using a double beam Ultraviolet-VIS spectrophotometer (Shimadzu, Japan) maintaining the wavelength at $227 \mathrm{~nm}$. The following formula was used for estimating the \% DEE [24].

$$
\mathrm{DEE} \%=\frac{\text { Actual drug content in microcapsules }}{\text { Theoretical drug content in microcapsules }} \times 100
$$

\section{Swelling characteristics of microspheres}

The swelling behaviour of microspheres of MSB-Starch alginate loaded with gliclazide was studied in two different media. The current study was done in $0.1 \mathrm{~N} \mathrm{HCl}$ of $\mathrm{pH} 1.2$ and $\mathrm{PBS}$ of $\mathrm{pH}$ 7.4. This methodology involves the addition of $100 \mathrm{mg}$ of microspheres to the bowl of the USP dissolution apparatus. Following the addition of microspheres, the respective media of about $500 \mathrm{ml}$ was added, and the microspheres were allowed to swell. The temperature condition of the apparatus was fixed at $37 \pm 0.5{ }^{\circ} \mathrm{C}$ with a paddle speed of $50 \mathrm{rpm}$ [24]. The estimation of swelling behaviour was done by removing the swollen microspheres at regular intervals, then drying the surface with tissue paper and weighing it. The following formula was utilized to calculate the swelling index of the above microspheres maintained in two different mediums.

$$
\% \text { Swelling }=\frac{\text { Weight of Microspheres after Swelling }- \text { Weight of Dry Micropheres }}{\text { Weight of dry Micropheres }} \times 100
$$

\section{Bioadhesion study}

The mucoadhesive nature of the prepared microsphere was assessed utilizing the in vitro wash off technique. The above study involves the utilization of freshly collected and excised intestinal mucosa of goat having dimensions of $2 \times 2 \mathrm{~cm}$. This excise piece of intestinal mucosa that was collected from a local slaughter house was than subjected to mounting on a clean glass slide $(7.5 \times 2.5 \mathrm{~cm})$ by attachment with a thread. The study involves the spreading of about 100 microspheres gently on to the wet and ringed specimen of tissue assembled on to a disintegration test apparatus to allow the continuous up and down movement. The entire study was carried out by using $900 \mathrm{ml} \mathrm{PBS}$ solution of $\mathrm{pH} 1.2$ with temperature maintained at $37 \pm 0.5^{\circ} \mathrm{C}$. At multiple time intervals, the count for the number of microspheres still attaching to the tissue specimen was done [24].

\section{Surface morphology by Field Emission Scanning Electron Microscopy (FESEM)}

The investigation of the morphology of the prepared microsphere was carried out by utilizing scanning electron microscopy (FESEM-S 4800, Hitachi, Japan) by maintaining the working distance of $8.6-8.8 \mathrm{~mm}$ and also keeping the accelerating voltage of $1.0 \mathrm{kV}$. The prepared microspheres were routinely examined for its shape, size and its surface characteristics. [25]

\section{In vitro release study}

The amount of drug released from the prepared microsphere was studied using an eight-station paddle type (USP Type II) dissolution apparatus (Lab India) each consisting of $900 \mathrm{ml}$ PBS solution of $\mathrm{pH} 7.4$ with the bath temperature and stirring speed maintained at $37 \pm 1{ }^{\circ} \mathrm{C}$ and $50 \mathrm{rpm}$, respectively. It is worth to be mentioned that gliclazide degrades in the gastric $\mathrm{pH}(1.2)$ and shows to be more soluble in the alkaline $\mathrm{pH}$ value as reported by Bansal et al. [26]. However, the drug remains in the stomach for only $2 \mathrm{~h}$; duration of time the drug proves to be stable. Consequently, the dissolution medium was chosen as PBS solution pH 7.4 as documented in BP 2013. An equivalent to $100 \mathrm{mg}$ of microsphere was taken and added to the six bowls of the dissolution test apparatus, whereas the remaining two bowls were left alone with only the buffer solution as the replacing fluid. At specific time interval, approximately $5 \mathrm{ml}$ of aliquot was collected and subsequently the same volume was replaced with fresh buffer in order to maintain the sink condition thought the entire experimental process. The collected aliquots were filtered and diluted, and estimation for GLZ content was done by using a UV-VIS double beam spectrophotometer (Shimadzu, Japan) at $227 \mathrm{~nm}$ wavelength [27].

\section{Kinetics of drug release}

The release kinetics serves as a useful tool in correlating both the response obtained from in vitro as well as in vivo analysis. This correlation is done mostly by comparing the pharmacokinetics results with that of the dissolution profile of the formulations. There are several available 
mathematical models like zero order, first order, Higuchi that helps in predicting the kinetics of drug release. To study the release kinetics of GLZ, DD solver (2010), an add-in program for Microsoft excels used for the purpose of modelling and comparison of drug release profile was used. [28]

\section{Drug release mechanism}

For evaluation of the drug release mechanism from the prepared microspheres, the data obtained from the in vitro drug release was plotted in Korsmeyer-Peppas equation [29] by taking log cumulative percent of drug release on $\mathrm{Y}$-axis and log time on $\mathrm{X}$-axis. The exponent " $n$ " was calculated through the slope of the straight line. The equation is

$$
\mathrm{M}_{\mathrm{t}} / \mathrm{M}_{\infty}=\mathrm{Kt}^{\mathrm{n}}
$$

Here, "Mt/M $\infty$ " indicates the fractional solute release, " $t$ " is the indication of time taken to release and the value of " $K$ " gives a representation of the kinetic constant characteristics in the drug/polymer system. The mechanism of drug release is mostly characterized by the exponent " $n$ " in the equation, and its value is significant in predicting the drug release pattern. If the exponent " $n$ " has a value that is 0.5 or less, the release mechanism mostly follows the Fickian diffusion. Similarly, if $0.5<\mathrm{n}<1$, the release mechanism mostly follows the non-Fickian or the anomalous diffusion. However, in case the exponent $n$ has a value greater than 1 , it mostly indicates the release pattern to be super case-II transport mechanism.

\section{In vivo testing of the optimized formulation}

The evaluation of the optimized batch of the prepared mucoadhesive microsphere was carried out by adopting several in vivo tests. The evaluation process included the measurement of pharmacokinetic parameters after single oral dose administration, and also pharmacodynamics evaluation of the prepared microsphere on streptozotocin-induced diabetic rats. Moreover, for the assessment of gastro-retentive behaviour, the technique of gamma scintigraphy was employed.

\section{Experimental animal selection and ethical consideration}

Healthy New Zealand white rabbits (weighing from $1.5-2 \mathrm{~kg}$ ) and albino Wistar rats (weighing from 150 to $200 \mathrm{~g}$ ) of either sex were procured from M/S Chakraborty Enterprise, Kolkata, West Bengal, India, and were used for the assessment of pharmacokinetics study, in vivo gastro-retentive behaviour and antidiabetic study. All the animals were housed in poly-acrylic cages having an assembly of wire mesh at the top along with a hygienic bed of husk in a specified animal room free from pathogen by maintaining the standard laboratory conditions (temperature $25 \pm 2{ }^{\circ} \mathrm{C}$ and relative humidity maintained at $50 \pm 10 \%)$ with light/ dark cycle $(12 / 12 \mathrm{~h})$ allowing free access to standard rat feed (VRK Nutritional Solution, Pune, India) and water. The study was approved (approval number- NIPS/AH/20/19) by the Institutional Animal Ethical Committee (IAEC) of NETES Institute of Pharmaceutical Science (NIPS), Kamrup, Assam, India, and was conducted in accordance with the committee for the purpose of control and supervision of experiments on animals (CPCSEA).

\section{Pharmacokinetic study}

For the pharmacokinetic study, the overnight fasten rats were divided into two groups with 5 in each $(n=5)$ in a parallel design. The first group was administered with the optimized batch of microsphere, whereas the second group received raw gliclazide powder all in a dose corresponding to $10 \mathrm{mg} / \mathrm{kg}$ gliclazide as such dose was previously reported in the literature. [30]

The doses were dispersed in $2 \mathrm{ml}$ of distilled water and were vortexed for $10 \mathrm{~s}$ not more than $1 \mathrm{~min}$ before oral administration. This was directly followed by adding $1 \mathrm{ml}$ of glucose solution in a dose of $1 \mathrm{~g} / \mathrm{kg}$ in order to prevent severe hypoglycaemia that might arise due to gliclazide administration.

A sample of $0.5 \mathrm{~mL}$ blood was collected from every rat into an EDTA tube by retro-orbital vein puncture at the following intervals: 0 (pre-dose), 0.5, 1, 2, 3, 4, 6 and $24 \mathrm{~h}$.

\section{Preparation of plasma samples}

The collected blood samples were centrifuged at $8000 \mathrm{rpm}$ for $20 \mathrm{~min}$ in order to separate out the plasma. The plasma that was separated was collected and stored at $-20{ }^{\circ} \mathrm{C}$ for further analysis purpose. The working standard phenytoin $(100 \mu \mathrm{L})$ having a concentration of $12 \mu \mathrm{g} / \mathrm{ml}$ was added to $100 \mu \mathrm{l}$ of plasma followed by vortexing for $30 \mathrm{~s}$ and continuing centrifugation at $10,000 \mathrm{rpm}$ for $15 \mathrm{~min}$ at $20{ }^{\circ} \mathrm{C}$ to allow plasma protein separation. Finally, the supernatant was filtered using a $0.2 \mu \mathrm{m}$ syringe filter and was injected into HPLC system. For each of the sample, GLZ/PHY peak area ratio was recorded and the concentration of GLZ was determined using the calibration curve constructed in plasma. [30]

\section{HPLC analysis of GLZ in rat plasmas}

The estimation of GLZ concentration in rat plasma was carried out using the same HPLC method as used in the in vitro quantification by using phenytoin (PHY) as the internal standard.

\section{Pharmacokinetic parameters}

The obtained data were further analysed for pharmacokinetic parameters by using PK solver (2010), an add-in 
program for Microsoft Excel used in analysis of pharmacokinetic and pharmacodynamics data. The area under the plasma concentration time profile $\left(\mathrm{AUC}_{0-24}\right.$ and $\mathrm{AUC}$ 0-inf) was calculated using the linear trapezoidal method. Also, the maximum plasma concentration $\left(C_{\max }\right)$ and the time to reach the maximum plasma concentration $\left(T_{\max }\right)$ were also determined subsequently [30].

\section{Pharmacodynamics study protocol In vivo anti diabetic study}

To access the antidiabetic activity of the prepared mucoadhesive microsphere, streptozotocin (STZ)induced diabetic rat model was used [30]. For the study purpose, diabetes was induced in the rats by intraperitoneal injection of freshly prepared streptozotocin (STZ) at a dose of $50 \mathrm{mg} / \mathrm{kg}$ body weight [31] dissolved in $0.1 \mathrm{M}$ cold sodium citrate buffer solution of $\mathrm{pH}$ 4.5. In order to avoid fatal hypoglycemia following administration of STZ, the animals were permitted to drink $5 \%(\mathrm{w} / \mathrm{v})$ glucose solution for the next $24 \mathrm{~h}$. After 3 days of STZ administration, a drop of blood was collected from tail vein from fasted rats for estimation of blood glucose level (Accu-Chek Extra Care Roche Diabetes Care India Pvt. Ltd.). The animals with serum glucose level more than $250 \mathrm{mg} / \mathrm{dl}$ were measured as diabetic and considered for further study. The rats were randomly divided into four groups with six in each $(n=6)$ as mentioned below.

Group I: Normal control rats (NC); received intraperitoneal injection of $0.9 \% \mathrm{w} / \mathrm{v} \mathrm{NaCl}$ solution $(1 \mathrm{ml} / \mathrm{kg}$ b.w. i.p.).

Group II: Diabetogenic control (STZ); received a single dose of streptozotocin (STZ).

Group III: Standard treatment $(\mathrm{STZ}+\mathrm{GL})$; following the STZ injection, this group received Glibenclamide at a dose of $5 \mathrm{mg} / \mathrm{kg}$ [32] b.w., p.o for the next three weeks.

Group IV: Test compound (STZ+GLM); alike to group III except that the test drug Gliclazide-loaded microsphere (10 $\mathrm{mg} / \mathrm{kg}$ b.w., p.o) [33] replaced the Glibenclamide.

The blood glucose level was estimated in all groups, before and after treatment on $3^{\text {rd }}, 10^{\text {th }}, 17^{\text {th }}$ and $24^{\text {th }}$ day from the overnight fasted rats. Blood samples were collected by retro-orbital venous plexus puncture method lipoprotein cholesterol, low-density lipoprotein cholesterol). All the biochemical parameters were measured using standard commercial kits with the help of a semi-auto-analyser (Erba Chem 7).

All the animals (rabbits and rats) used in both the studies were not subjected to euthanasia since none of the animals was killed after completion of the experimental period. However, post-experimental and after a definite wash out period all the animals were further used for monitoring behavioural changes.

\section{Pharmacoscintigraphic study}

To estimate the in vivo gastro-retentive behaviour of the prepared mucoadhesive gliclazide microsphere following oral administration and determine its extent of transit through the GI tract, the gamma scintigraphy technique was used [34]. The overnight fastened rabbits were randomly divided into two groups with 3 in each $(n=3)$ with one serving as the control group. The radio labelling technique for the prepared microsphere was carried out in a stannous chloride that is a potent reducing agent. This radio labelling technique with $99 \mathrm{mTc}$-pertechnetate involves $3 \mathrm{mg}$ of the gliclazide-loaded mucoadhesive microsphere in a glass vial, which was previously sterilized following the addition of $1 \mathrm{ml}$ of distilled water to it. This was followed by the addition of reducing agents of concentrations about $60 \mu \mathrm{g}$ and maintaining the $\mathrm{pH}$ of the solution to 7.5. Finally, filtration was carried out for the above contents through a Whatman filter paper (\#41) into a sterile vial, and an approximate of about $18.5 \mathrm{MBq}$ 99mTc-pertechnetate was added, mixed and incubated for about 5-10 min. Furthermore, to determine the radio labelling efficiency of the complex, the technique of paper chromatography was used. The mobile phase used in this process was acetone (100\%). The spotting of the sample was done initially after labelling the microsphere and before the washing step. The pertechnetate tends to migrate to the crest of the stationary phase, whereas the microsphere-attached material tends to remain at the initial point of application. The labelling yield here was expressed as a percentage of the total amount of radioactivity applied in the testing system, calculated using the formula.

$$
\text { Radio labelling }=\frac{\text { Radioactivity (Counts) Retain in the Lower Half of strip }}{\text { Initial Radioactivity (Total Count Present) with the Strip }} \times 100
$$

under mild ether anaesthesia. Blood was allowed to stand for $10 \mathrm{~min}$ at room temperature, centrifuged at $2500 \mathrm{rpm}$ for $10 \mathrm{~min}$ to separate the serum for assessment of lipid profile (Triglycerides, Total Cholesterol, High-density
To restrict the movement of the animals during imaging, the four legs of the animals were tied to plywood. The radio-labelled formulation was finally administered to the animal of the first group via a feeding tube followed 
by drinking water in sufficient volume through the same tube. The animals were subjected to imaging with the help of a gamma camera (Siemens AG, Munich, Germany) to locate the formulation. Scanning of the whole anterior body of the rabbit was carried out for $15 \mathrm{~min}$, and the $140 \mathrm{keV}$ gamma rays that were emitted by $99 \mathrm{mTc}$ were imaged. The gamma camera used single photon emission computed tomography (SPECT) to detect the amount of emission. The gamma images generated were recorded by utilization of an online computer system.

\section{Statistical analysis}

The determination of statistical significance was carried out using the statistical software package Graph Pad Prism version 6.0 (Graph Pad Software, San Diego, CA, USA). The results are expressed as mean \pm SEM, and the results were analysed using a one-way ANOVA test. Further, the Dunnett's multiple comparison tests was followed considering the $\mathrm{p}$ values of $<0.05$ as significant.

\section{Results}

\section{Isolation and identification starch and preparation} of microsphere

The starch used for the current study was isolated from the fruits of Musa balbisiana (MSB-Starch) followed by some physical and chemical modifications. The starch obtained was confirmed from the positive results in terms of colour observed after addition of iodine. The overall yield of the starch collected from the fruits was found to be about $54 \%$. Following the collection of starch, the microsphere was prepared by using the technique of Ionic gelation. In total, nine batches of gliclazide-loaded mucoadhesive microsphere were prepared to vary the concentration of the MSB-Starch and Na-alginate and also varying the concentration of cross-linking agent $\mathrm{CaCl}_{2}$.

\section{Formulation optimization}

In the current $3^{2}$ factorial design, Polymer (MSB Starch: sodium alginate) and cross-linking agent $\left(\mathrm{CaCl}_{2}\right)$ were selected as the independent variable having three levels $+1,0,-1$ for high, intermediate and low, respectively. In total, 9 different formulations were prepared by altering the ratio of polymer and cross-linking agents and the results obtained by evaluation of the prepared formulation were finally added to the software in order to achieve the results for model significance. The obtained responses in terms of \% Drug loading, \% Bioadhesion and \% Drug release were recorded for all the prepared nine formulations. The obtained response data are shown in Table 1.

\section{Drug loading study}

After application of factorial design, suggestion for a linear model was given by the software for the response Y1 (Drug loading). The significance of this effect was further validated with the model Fischer's (F) value of 111.98 and $\mathrm{p}$ value $<0.0500$ indicating it. Furthermore, the linear model was significant and only a $0.01 \%$ probability that this larger value of $\mathrm{F}$ could be because of noise. Moreover, the values of Predicted $R^{2}(0.9404)$ resides in a reasonable agreement with that of the values of Adjusted $\mathrm{R}^{2}$ (0.9652), and as the difference is less than 0.2, it indicates the model to be fit. The "Adeq Precision" gives the measurement of signal to the noise ratio and a ratio which is greater than 4 is mostly desirable. Here, the ratio obtained is 26.186 which gives an indication of an adequate signal thereby providing the reliability of the model's utility in navigation of the design space Table 2a [35-38]

Table 1 Response parameters

\begin{tabular}{|c|c|c|c|c|c|c|}
\hline Std & Run & $\begin{array}{l}\text { Factor } 1(\mathrm{X} 1) \\
\text { A: polymer } \\
\text { gm }\end{array}$ & $\begin{array}{l}\text { Factor } 2(X 2) \\
\text { B: cross-linking agent } \\
\text { gm }\end{array}$ & $\begin{array}{l}\text { Response } 1 \\
\text { DL } \\
\%\end{array}$ & $\begin{array}{l}\text { Response } 2 \\
\text { BA } \\
\%\end{array}$ & $\begin{array}{l}\text { Response } 3 \\
\text { DR } \\
\%\end{array}$ \\
\hline 1 & 1 & $3(-1)$ & $2(-1)$ & 63.77 & 69.12 & 97.44 \\
\hline 9 & 2 & $9(+1)$ & $6(+1)$ & 75.42 & 80.10 & 76.87 \\
\hline 8 & 3 & $6(0)$ & $6(+1)$ & 79.37 & 82.13 & 78.87 \\
\hline 6 & 4 & $9(+1)$ & $4(0)$ & 70.12 & 86.22 & 75.40 \\
\hline 2 & 5 & $6(0)$ & $2(-1)$ & 60.23 & 73.19 & 90.55 \\
\hline 4 & 6 & $3(-1)$ & $4(0)$ & 74 & 72.11 & 88.66 \\
\hline 5 & 7 & $6(0)$ & $4(0)$ & 71.40 & 78.10 & 81.10 \\
\hline 7 & 8 & $3(-1)$ & $6(+1)$ & 80.72 & 76.50 & 81.20 \\
\hline 3 & 9 & $9(+1)$ & $2(-1)$ & 57.40 & 81.50 & 86.25 \\
\hline
\end{tabular}

Polymer: Musa balbisiana starch: Sodium alginate (1:1 in all formulation); Cross-linking agent: Calcium chloride; DL: Drug Loading; BA: Bio adhesion; DR: Drug release Coded values are in bracket; $(X 1)$ and $(X 2)$ are independent factors; $(+1)=$ higher values; $(0)=$ medium value and $(-1)=$ lower values 
Table 2 Model statistics for responses

\begin{tabular}{|c|c|c|c|c|c|c|}
\hline \multicolumn{7}{|c|}{ ANOVA of dependant variables } \\
\hline Dependant variables & Source of variables & Sum of squares & $\begin{array}{l}\text { Degree of } \\
\text { freedom }\end{array}$ & Mean square & F value & P value \\
\hline Drug loading & $\begin{array}{l}\text { Regression } \\
\text { Residuals } \\
\text { Total } \\
\mathrm{R}^{2}\end{array}$ & $\begin{array}{l}528.28 \\
14.15 \\
542.44 \\
0.9739\end{array}$ & $\begin{array}{l}2 \\
6 \\
8\end{array}$ & $\begin{array}{r}264.14 \\
2.36\end{array}$ & 111.98 & $<0.0001$ Significant \\
\hline Bio adhesion & $\begin{array}{l}\text { Regression } \\
\text { Residuals } \\
\text { Total } \\
\mathrm{R}^{2}\end{array}$ & $\begin{array}{l}188.00 \\
51.21 \\
239.21 \\
0.7859\end{array}$ & $\begin{array}{l}2 \\
6 \\
8\end{array}$ & $\begin{array}{r}94.00 \\
8.53\end{array}$ & 11.01 & 0.0098 Significant \\
\hline Drug release & $\begin{array}{l}\text { Regression } \\
\text { Residuals } \\
\text { Total } \\
\mathrm{R}^{2}\end{array}$ & $\begin{array}{l}369.93 \\
47.74 \\
471.67 \\
0.8857\end{array}$ & $\begin{array}{l}2 \\
6 \\
8\end{array}$ & $\begin{array}{r}184.96 \\
7.96\end{array}$ & 23.25 & 0.0015 Significant \\
\hline \multicolumn{7}{|l|}{ Model statistics } \\
\hline Model $\mathrm{R}^{2}$ & $R^{2}$ & Adjusted $\mathrm{R}^{2}$ & Predicted $\mathrm{R}^{2}$ & PRESS & s.d & Remarks \\
\hline \multicolumn{7}{|c|}{ Response $Y_{1}$ (drug loading) } \\
\hline Linear & 0.9739 & 0.9652 & 0.9404 & 32.35 & 1.54 & Suggested \\
\hline Interactive & 0.9744 & 0.9591 & 0.8831 & 63.41 & 1.67 & \\
\hline Quadratic & 0.9949 & 0.9864 & 0.9430 & 30.90 & 0.9587 & \\
\hline \multicolumn{7}{|c|}{ Response $Y_{3}$ (drug release) } \\
\hline Linear & 0.8857 & 0.8476 & 0.7211 & 116.49 & 2.82 & Suggested \\
\hline Interactive & 0.9139 & 0.8622 & 0.6090 & 163.30 & 2.68 & \\
\hline Quadratic & 0.9748 & 0.9328 & 0.6929 & 128.25 & 1.87 & \\
\hline
\end{tabular}

The polynomial equation for response $Y_{1}$ is as follows

$$
Y_{1}=+57.41667-0.863889 X_{1}+4.50917 X_{2}
$$

\section{Bioadhesion study}

After application of factorial design, suggestion for a linear model was given by the software also for the response Y2 (Bioadhesion study). The significance of this effect was further validated with the model Fischer's value $(F)$ value of 11.01 and p value 0.0500 indicating it. Furthermore, the linear model was significant and only a $0.98 \%$ probability that this larger value of F could be because of noise. Moreover, the values of Predicted $R^{2}(0.4225)$ is not as close with that of the values of Adjusted $R^{2}(0.7146)$, and as the difference is more than 0.2 , it indicates possibilities of a large block effect or more possibilities with the model and/or data. The things that are to be considered here are model reductions, outliers, repose transformation, etc. [35-38].

The "Adeq Precision" gives the measurement of signal to the noise ratio and a ratio which is greater than 4 is mostly desirable. Here, the ratio obtained is 8.895 which give an indication of an adequate signal thereby providing the reliability of the model's utility in navigation of the design space Table $2 \mathrm{a}$

The polynomial equation for response $Y_{2}$ is as follows

$$
Y_{2}=77.66+5.02 X_{1}+2.49 X_{2}
$$

\section{Drug release study}

After application of factorial design, suggestion for a linear model was given by the software also for the response Y3 (Drug release study). The significance of this effect was further validated with the model Fischer's $(F)$ value of 23.25 and p value 0.0015 indicating it. Furthermore, the linear model was found to be significant and there is only a $0.15 \%$ probability that this larger value of F could be because of noise. Moreover, the values of Predicted $R^{2}(0.7211)$ resides in a reasonable agreement with that of the values of Adjusted $R^{2}$ (0.8476), and as the difference is less than 0.2 , it indicates the model to be fit. The "Adeq Precision" gives the measurement of signal to the noise ratio and a ratio which is greater than 4 is mostly desirable. Here, the ratio is 13.526 which give an indication of an adequate signal thereby providing the reliability of the model's utility in navigation of the design space Table $2 \mathrm{a}$ [35-38]

The polynomial equation for response $Y_{3}$ is as follows

$$
Y_{3}=+106.06444-1.59889 X_{1}-3.10833 X_{2}
$$




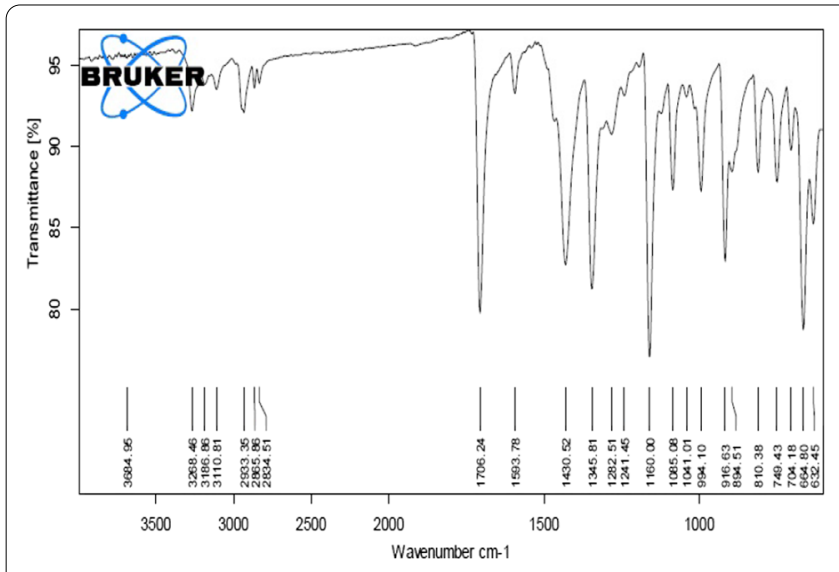

$1 \mathrm{~A}$

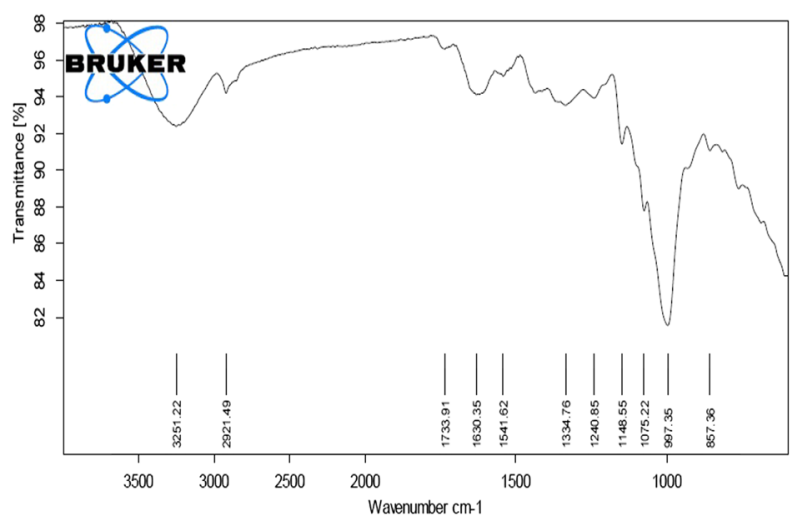

$1 \mathrm{C}$

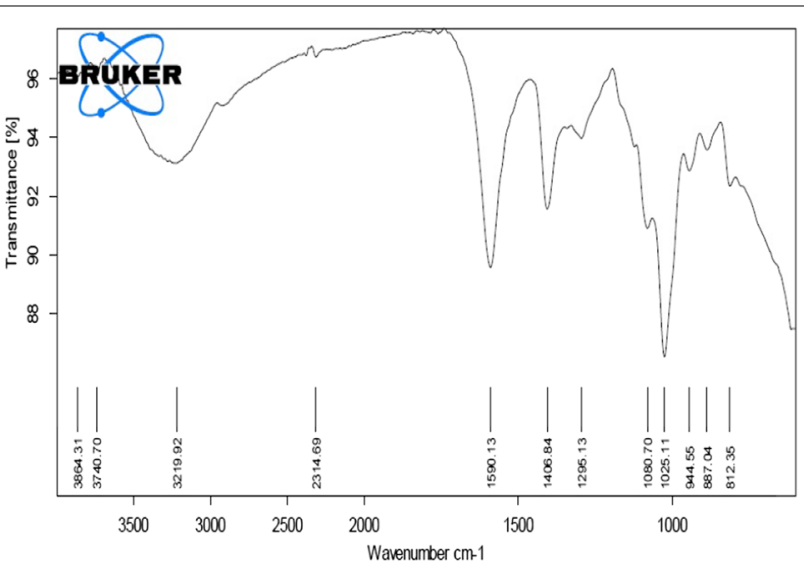

1B

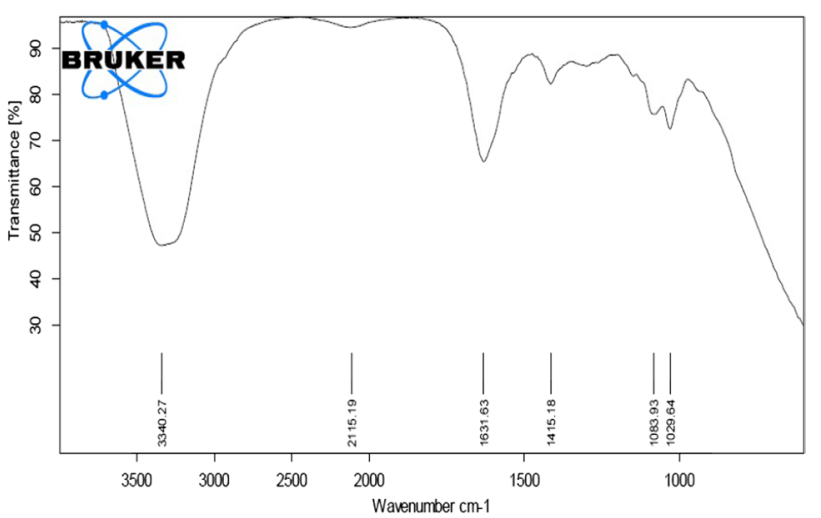

1D

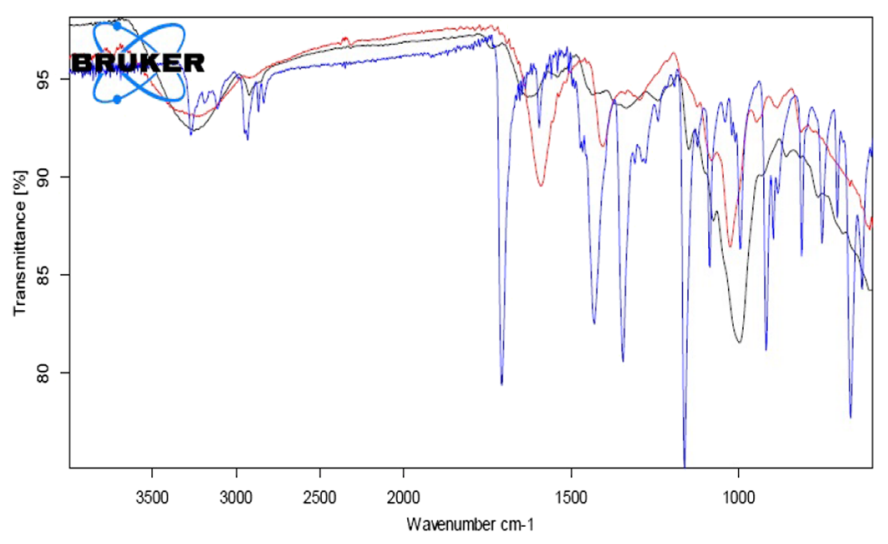

$1 \mathrm{E}$

Fig. 1 The FTIR-spectra are shown here. The spectra obtained after scanning from 4000 to $400 \mathrm{~cm}^{-1}$ and maintaining the resolution at $4 \mathrm{~cm}^{-1}$ show the presence of prominent functional groups $\mathbf{A}$ FTIR — spectra of pure gliclazide $\mathbf{B}$ FTIR — spectra of sodium alginate $\mathbf{C}$ FTIR - spectra of starch of Musa balbisiana species D FTIR—spectra of drug-loaded microsphere $\mathbf{E}$ overlap FTIR—spectra of pure drug, starch and sodium alginate

\section{Drug and excipients compatibility study}

The absence of any possible interaction between the drug and the excipients was established from the results obtained from FTIR study, DSC study and IST studies.
There was no any development of new peak for the drug polymer mixture when compared with the FTIR spectra for pure drug and excipients. Similarly, the DSC and IST study data (Table 3) also provided an evidence of the 


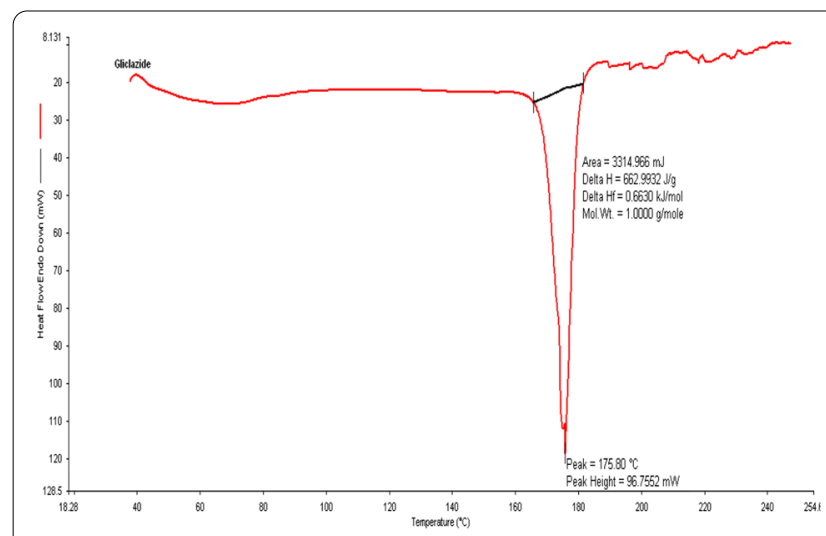

2 A

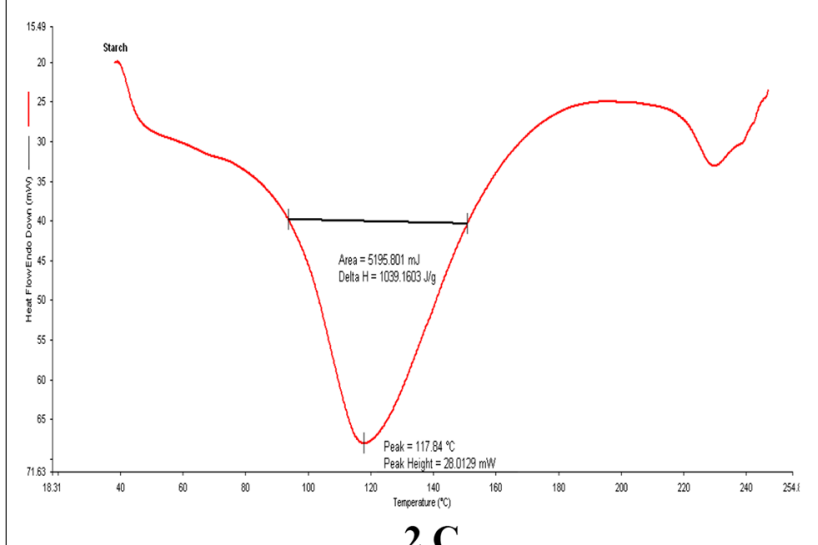

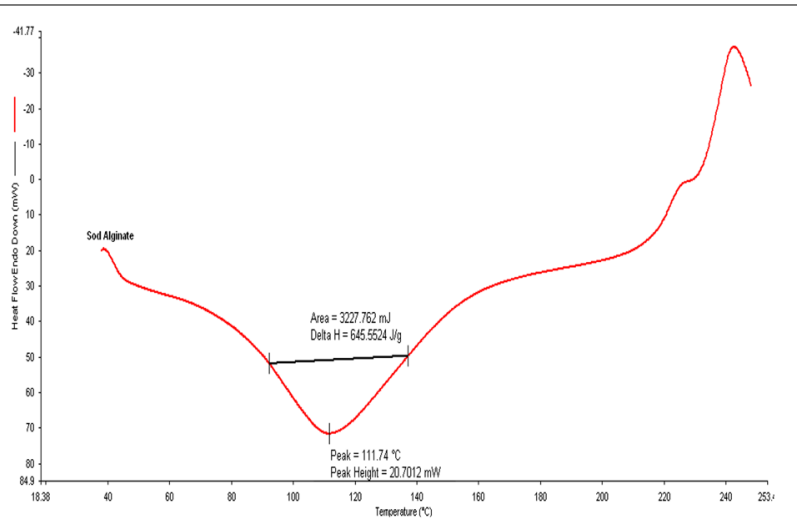

2B

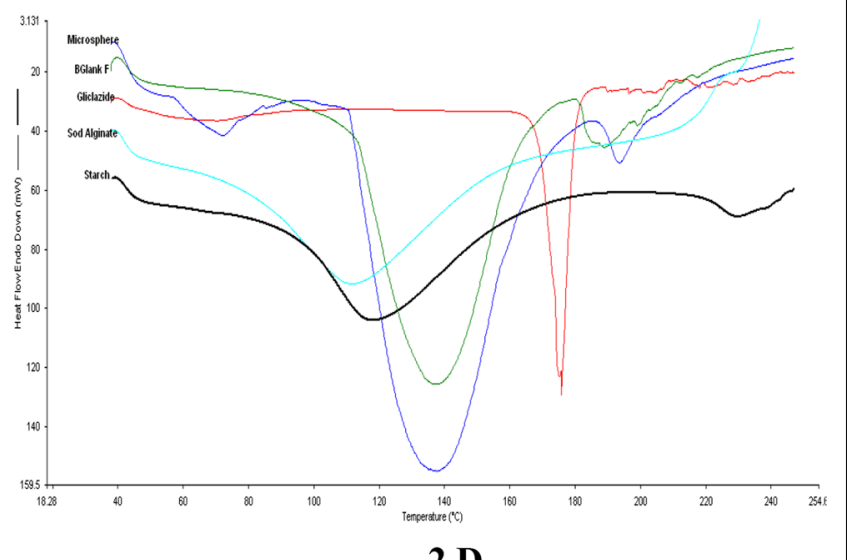

$2 \mathrm{D}$

Fig. 2 The DSC thermogram is shown here $\mathbf{A}$ DSC thermogram of pure gliclazide $\mathbf{B}$ DSC thermogram of sodium alginate $\mathbf{C}$ DSC spectrum of starch obtained from Musa balbisiana species D DSC thermogram of Drug-loaded microsphere, blank microsphere, pure gliclazide, sodium alginate and starch

Table 3 Results of isothermal stress testing of pure drug and drug excipient mixture after 3 weeks of storage

\begin{tabular}{|c|c|c|c|c|c|}
\hline \multirow[t]{2}{*}{ Sample } & \multirow{2}{*}{$\begin{array}{l}\text { Ratio (drug: } \\
\text { excipients) }\end{array}$} & \multicolumn{2}{|c|}{ Quality unchanged $(\% w / w) *$} & \multicolumn{2}{|c|}{ Wavelength maxima ( $\lambda$ max) } \\
\hline & & Control & Stressed & Control & Stressed \\
\hline GLZ (pure) & - & $100.55 \pm 0.089$ & $99.42 \pm 0.170$ & 227 & 227 \\
\hline GLZ + sodium alginate & $2: 2$ & $100.37 \pm 0.251$ & $99.29 \pm 0.266$ & 227 & 227 \\
\hline GLZ+MSB starch & $2: 2$ & $100.44 \pm 0.060$ & $99.31 \pm 0.077$ & 227 & 227 \\
\hline
\end{tabular}

*Mean \pm S.D $(\mathrm{n}=3)$

GLZ: Gliclazide; MSB: Musa balbisiana starch

stable nature of the drug and the excipients used in the current study (Figs. 1, 2).

\section{Production yield}

The yields of production for the microsphere formulated by using the technique of ionic gelation were found to be in the range between 70 and $88 \%$.
Flow property study of prepared microsphere

The free flowing nature of the prepared microsphere was established from the results obtained from angle of repose, car's index and Hausner ratio study. The data for the same are represented in Table 4 
Table 4 Flow property study of prepared microsphere

\begin{tabular}{|c|c|c|c|c|c|}
\hline Sl. no. & Formulation code & Angle of repose $(\varnothing)^{*}$ & Carr's index* & Hausner's ratio & $\begin{array}{l}\text { True density } \\
\left(\mathrm{g} / \mathrm{cm}^{3}\right)^{*}\end{array}$ \\
\hline 1 & F1 & $24.73 \pm 0.124$ & $96.29 \pm 0.040$ & 0.03 & $0.82 \pm 0.011$ \\
\hline 2 & F2 & $25.50 \pm 0.216$ & $96.37 \pm 0.055$ & 0.04 & $0.83 \pm 0.01$ \\
\hline 3 & F3 & $24.50 \pm 0.294$ & $96.41 \pm 0.130$ & 0.03 & $0.86 \pm 0.02$ \\
\hline 4 & F4 & $24.56 \pm 0.339$ & $96.41 \pm 0.005$ & 0.03 & $0.85 \pm 0.005$ \\
\hline 5 & F5 & $24.73 \pm 0.385$ & $96.30 \pm 0.005$ & 0.04 & $0.90 \pm 0.005$ \\
\hline 6 & F6 & $24.30 \pm 0.355$ & $96.47 \pm 0.055$ & 0.04 & $0.92 \pm 0.005$ \\
\hline 7 & F7 & $24.46 \pm 0.262$ & $96.50 \pm 0.181$ & 0.04 & $0.90 \pm 0.005$ \\
\hline 8 & F8 & $24.96 \pm 0.169$ & $96.62 \pm 0.011$ & 0.03 & $0.91 \pm 0.011$ \\
\hline 9 & F9 & $25.23 \pm 0.124$ & $96.61 \pm 0.01$ & 0.03 & $0.92 \pm 0.01$ \\
\hline
\end{tabular}

${ }^{*}$ Mean $\pm S D(n=3)$

Drug loading and entrapment efficiency Efficiency of drug entrapment

The drug entrapment efficiency of the gliclazide-loaded Banana starch microsphere was found to be in the values as given in Table 1.

\section{Size analysis}

Size analysis of the microspheres when observed under was found to be in the range of $767.11 \pm 20.92 \mu \mathrm{m}-913.3$ $6 \pm 10.55 \mu \mathrm{m}$.

\section{Swelling behaviour}

The swelling behaviour of the prepared gliclazide-loaded mucoadhesive microsphere was carried out on both gastric and intestinal pH. As shown in Fig. 3A, B, the study data revealed that the swelling index was lower in $0.1 \mathrm{~N}$ $\mathrm{HCl}, \mathrm{pH} 1.2$ (gastric $\mathrm{pH}$ ) than PBS, pH 7.4 (intestinal pH).

\section{Mucoadhesive analysis}

The investigation of the mucoadhesiveness of the prepared microsphere was carried out by the in vitro washoff method that employs intestinal mucosa of goat in two $\mathrm{pH}$ environments. The $\mathrm{pH}$ of 1.2 replicates the gastric environment, whereas the $\mathrm{pH}$ of 7.4 replicates the intestinal environment as shown in Fig. 4A, B, respectively.

\section{In vitro release and kinetic study}

The release study from the prepared batches of GLZ microsphere was studied in PBS solution $\mathrm{pH} 7.4$ using the 8 station Type II dissolution apparatus. The release of the active medicament was estimated by collecting aliquot of samples at different time interval, and the estimation of the same for the active ingredient was done in a UV visible spectrophotometer at $227 \mathrm{~nm}$. The results for the same are shown in Table 5. The different batches of microspheres (F1-F9) showed a prolong release of gliclazide over a period of $12 \mathrm{~h}$. The in vitro drug release data of the optimized GLZ-loaded microsphere were evaluated kinetically using various mathematical models.

\section{Surface morphology by field emission scanning electron microscopy (FESEM)}

FESEM pictures (Fig. 5) obtained for the prepared microsphere demonstrated the surface characteristic of the formulation. There was development of smooth particles, and these are often favoured for the uptake of the dosage form through the cells and to the lymphatic tissues.

\section{Pharmacokinetic study}

The concentration of drug in the plasma sample that was collected was estimated by HPLC analysis. The calibration curve of GLZ was found to be linear over the range of $0.5-40 \mu \mathrm{g} / \mathrm{ml}$ with $\mathrm{R}^{2}$ value to be 0.9924 . Both the peak of GLZ and PHY separated at 1.9 and $3.4 \mathrm{~min}$, respectively, without any tailing and fronting. The estimation of accuracy was depicted by the \% recovery of GLZ that ranged from $94 \%-98 \%$. The intra-day and inter-day precision was presented by CV\% that ranged from $1.50 \%$ to 11.50. The mean plasma concentration-time profile is plotted in Fig. 6

\section{Gamma scintigraphy study for studying the gastro-retentive behaviour}

This technique of gamma scintigraphy utilizes the tagging of a short-lived gamma-emitting radioisotope that is a noninvasive procedure [39]. In this present study, $99 \mathrm{mTc}$-pertechnetate was utilized to tag with the optimized batch of the prepared mucoadhesive microsphere. The objective of this study was to provide a complete proof of concept justifying the bioadhesiveness of the dosage form. It was also seen that the neuron-activated microsphere produced no radioactive by-products. The utilization of hybrid imaging of SPECT and computed tomography (CT) was chosen because the technique of 


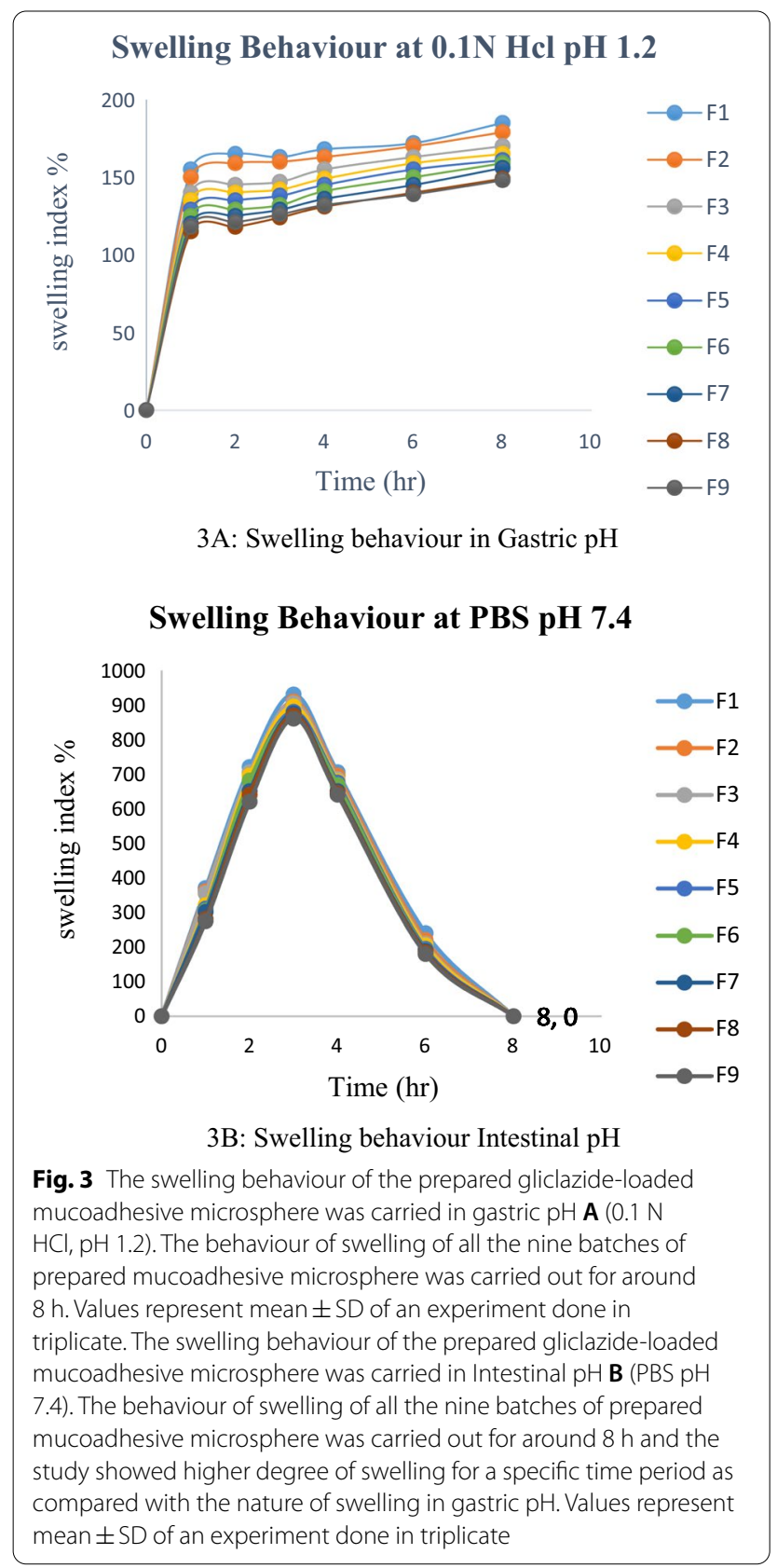

CT provides excellent images that give an idea of the anatomical information to provide identification of the organ structure.

The scintigrams provided useful information related to the transit time and also about the location of the formulation in the GI tract. The GI section was critically analysed in the gamma scintigraphy analysis, and there was a noticeable difference observed at $0 \mathrm{~h}$ and $1 \mathrm{~h}$ after oral administration as shown in Fig. 7A, B, respectively. The marker particles were mostly visible in dark colour dots that recognize the particulate. As seen in Fig. $7 \mathrm{C}$, the

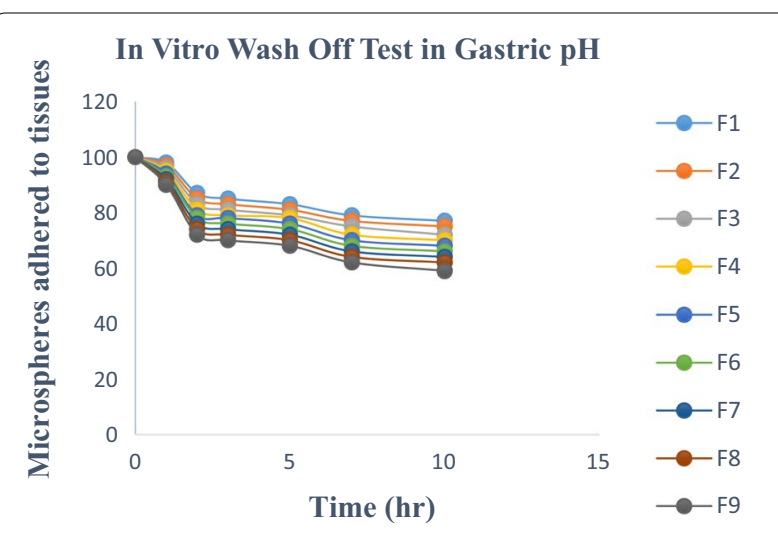

4A. In-vitro wash of test in Gastric $\mathrm{pH}$

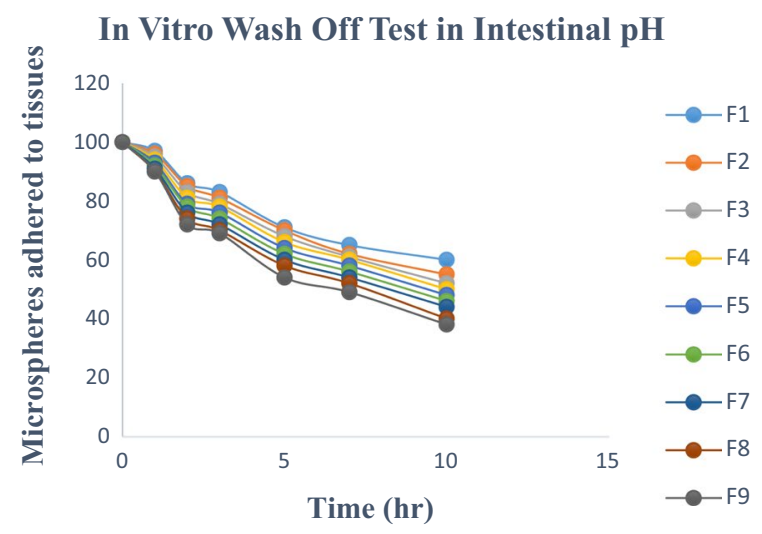

4B. In-vitro wash of test in intestinal $\mathrm{pH}$

Fig. 4 The in vitro wash off test of prepared microsphere was carried out in Gastric $\mathrm{pH} \mathbf{A}(0.1 \mathrm{~N} \mathrm{HCl}, \mathrm{pH} 1.2)$. The in vitro wash off test of prepared microsphere was carried out in Intestinal pH B (PBS pH 7.4). The wash off behaviour of all the nine batches of prepared mucoadhesive microsphere was carried out for around $10 \mathrm{~h}$

presence of the microsphere was marked in the stomach at $4 \mathrm{~h}$. However, post $2 \mathrm{~h}$, the formulation moved towards the small intestine as noticed in Fig. 7D for the gamma scintigraphy image at $6 \mathrm{~h}$ which reveals the in vivo gastro-retentive nature of the optimized formulation for almost a time period of $4 \mathrm{~h}$. Since the half-life of $99 \mathrm{mTc}-$ pertechnetate is around 5-6 h, there was a significant reduction in the \% radioactivity and therefore, the presence of the microsphere in the GIT could not be clearly assessed post $24 \mathrm{~h}$ of administration (Fig. 8).

\section{Antidiabetic study}

The in vivo efficiency of the optimized gliclazide-loaded mucoadhesive microsphere was performed in STZinduced diabetic rats and was estimated by measuring the blood glucose level. It was seen that there was an increase in the glucose level after STZ administration when compared with the normal group. As expected, 
Table 5 In vitro drug release study of the 9 batches of microspheres

\begin{tabular}{|c|c|c|c|c|c|c|c|c|c|}
\hline & F1 & F2 & F3 & F4 & F5 & F6 & F7 & F8 & F9 \\
\hline 0 & 0 & 0 & 0 & 0 & 0 & 0 & 0 & 0 & 0 \\
\hline 1 & 10.47 & 16.21 & 13.25 & 14.21 & 15.87 & 17.42 & 14.55 & 12.28 & 13.23 \\
\hline 2 & 13.92 & 21.42 & 16.67 & 19.5 & 18.27 & 25.93 & 21.53 & 21.34 & 19.45 \\
\hline 3 & 19.63 & 25.32 & 21.36 & 24.63 & 27.9 & 35.59 & 29.53 & 28.8 & 27.66 \\
\hline 4 & 24.2 & 31.82 & 24.68 & 32.8 & 32.62 & 47.46 & 39.34 & 39.54 & 38.48 \\
\hline 5 & 28.14 & 37.21 & 28.18 & 38.73 & 38.85 & 56.58 & 44.5 & 46.45 & 49.9 \\
\hline 6 & 35.81 & 43.81 & 34.41 & 43.21 & 43.17 & 60.22 & 52.26 & 54.32 & 55.65 \\
\hline 7 & 42.16 & 48.21 & 41.37 & 49.98 & 51.39 & 67.64 & 59.36 & 60.86 & 63.32 \\
\hline 8 & 48.33 & 54.31 & 47.54 & 54.72 & 60.42 & 75.27 & 64.66 & 68.88 & 69.19 \\
\hline 9 & 54.42 & 61.88 & 54.83 & 61.24 & 68.25 & 82.19 & 71.48 & 72.1 & 73.54 \\
\hline 10 & 59.56 & 67.21 & 62.74 & 66.65 & 74.76 & 87.33 & 83.4 & 75.58 & 76.55 \\
\hline 11 & 66.25 & 71.58 & 69.14 & 72.37 & 80.56 & 91.43 & 85.5 & 80.45 & 78.58 \\
\hline 12 & 75.4 & 78.87 & 76.87 & 81.1 & 86.25 & 97.44 & 90.55 & 88.66 & 81.2 \\
\hline
\end{tabular}

*Values are indicated as percent (\%) cumulative drug release
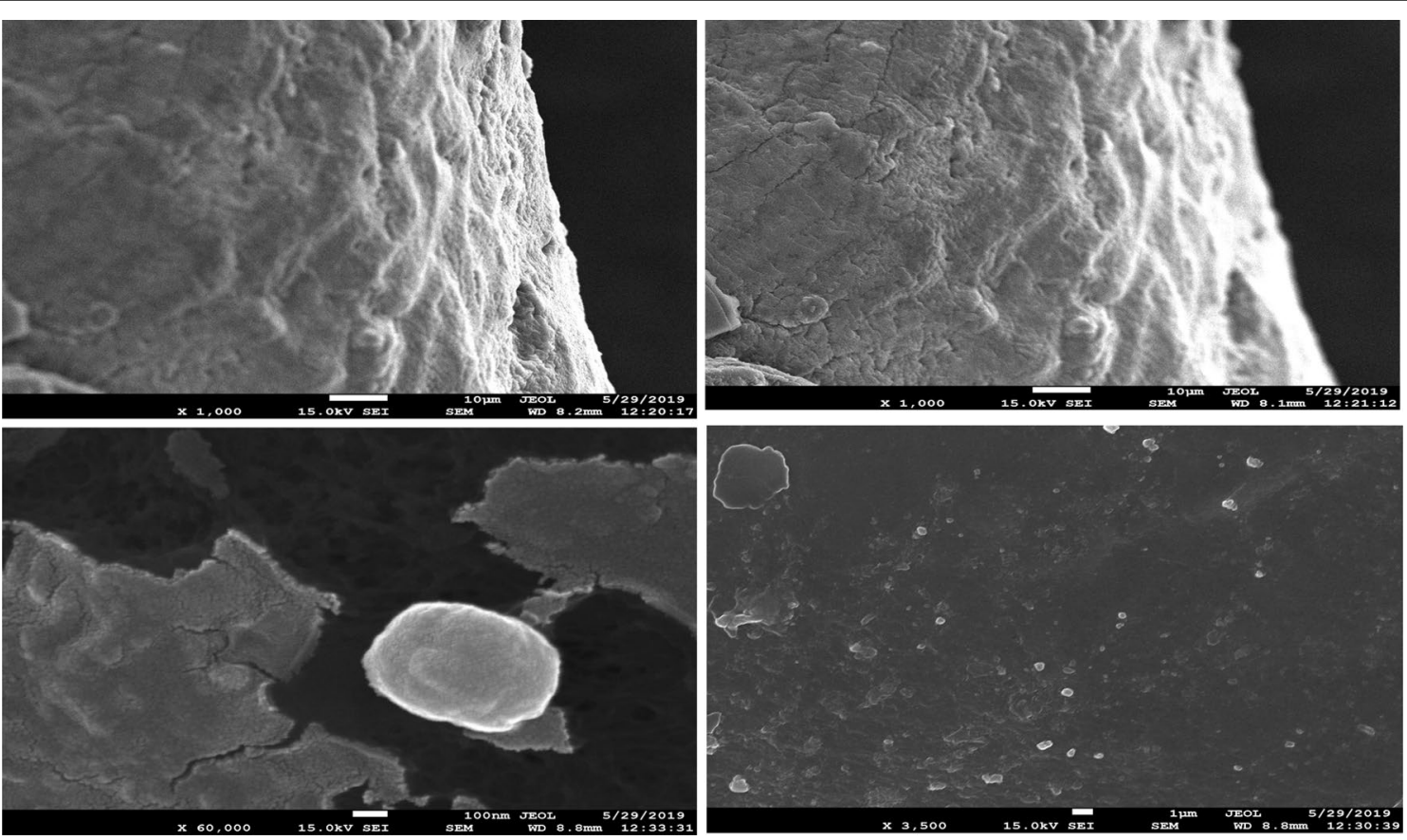

Fig. 5 FESEM photographs of the surface of the prepared mucoadhesive microsphere loaded with gliclazide. The image indicated smooth surface with occasional wrinkles and presence of polymeric debris

glibenclamide showed a decrease in blood glucose level when compared with the STZ group. Also, the optimized mucoadhesive microsphere loaded with gliclazide also showed a potential decrease in blood glucose level. The comparative in vivo blood glucose level along with the reduction in blood glucose level in STZ-induced diabetic rats after oral administration of the optimized gliclazide-loaded microsphere is shown in Fig. 9A. There was also an elevation of the total cholesterol level along with triglyceride level and LDL-c level in the STZ-treated group along with the lowering of HDL-c level as seen in the same group when compared with the normal group. Whereas, in the treatment group (both in the glibenclamide and gliclazide-loaded microsphere) there was an alteration in the level of lipid profile. The results for the same are given in Fig. 9B. 


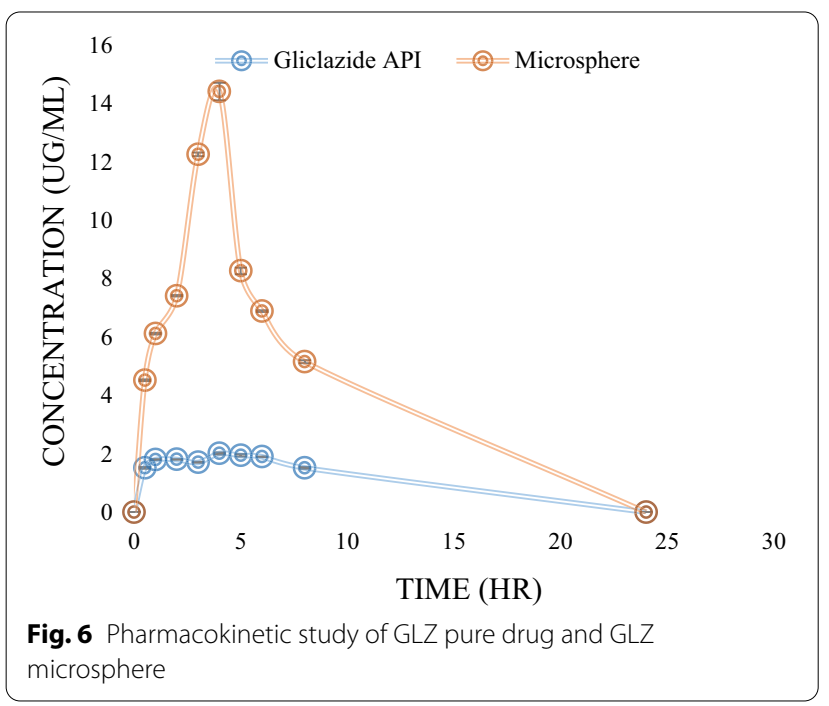

\section{Discussions}

The microsphere was prepared adopting the ionic gelation technique using the starch isolated form MSB species. The formation of spherical sized microspheres resulted in a good micrometrics property. The nature of free-flowing behaviour of the prepared microsphere can be predicted from the data's obtained from angle of repose where the values ranged from $24.3 \pm 0.355^{\circ}$ to $25.5 \pm 0.216^{\circ}$ indicating a good flow. The values for Carr's index, Hausner's ratio and true density were found to be around $96.29 \pm 0.40$ to $96.62 \pm 0.011,0.03-0.04$ and $0.82 \pm 0.011-0.92 \pm 0.01$, respectively, which also support the free-flowing nature of the prepared microspheres (Figs. 10, 11).

The microspheres obtained were found to have a discrete spherical shape and a good production yield. It was noted that the production yields of the formulations gradually decreased with the elevation in the concentration of the polymers. This can be related to the high viscosity of the polymer solution. The increase in viscosity of the solution results in blockage of the needle and causing wastage of the drug-polymer solution and ultimately reducing the production yield [40]. The enhancement of the size of microspheres can be related to the growth in viscosity of the polymeric solution as the concentration was elevated. This elevation in viscosity, in turn, increases the droplet size when the polymer-drug mixture was added to the cross-linking solution, thereby resulting in a larger size of particles. Moreover, as the concentration of the cross-linking agent $\left(\mathrm{CaCl}_{2}\right)$ was increased, a gradual decrease in the size of the microsphere was noticed. This may be because of the high degree of cross-linking with the polymer and cross-linker, resulting in compression of the polymeric gel, thereby decreasing its size. Figure 12

In case of estimating the drug entrapment efficiency, it was seen that as the ratio of polymer concentration increases, the entrapment efficiency decreases. The highest drug entrapment efficiency was seen as the polymer ratio was kept towards the lower side, and the concentration of the cross-linking agent was kept towards the higher side. This is thought to be because of the greater degree of cross-linking between sodium alginate and $\mathrm{CaCl}_{2}$. The presence of a high amount of cross-linker helps in converting sodium ions present in sodium alginate to $\mathrm{CaCl}_{2}$. This calcium alginate, in turn, helps in providing cross-linking tendency to form a cross-linked microsphere. However, with the low concentration of calcium chloride, the microspheres formed might have larger pores, resulting in minimal entrapment of the drug [41].

In swelling studies, the lower value of swelling index in gastric $\mathrm{pH}$ was due to the shrinkage of $\mathrm{Na}$-alginate in the acidic environment. Furthermore, the maximum swelling of the microsphere was at $2-3 \mathrm{~h}$ in the intestinal $\mathrm{pH}$, following which the erosion and finally dissolution process took place. Several reports suggested that this swelling behaviour of $\mathrm{Ca}++$ alginate was related to the cumulative translation of $\mathrm{Ca}++$ ions from calcium alginate [42]. Further reports also suggested that in intestinal $\mathrm{pH}$, the swelling might enhance because of phosphate ions in the PBS that act as a sequestering agent for calcium [43]. Hence, the nature of the swelling behaviour of MSB-Starch alginate loaded with gliclazide in PBS pH 7.4 can be related to the phenomenon of ion interchanging between the $\mathrm{Ca}++$ ions of the cross-linked microsphere

\footnotetext{
(See figure on next page.)

Fig. 7 Gamma Scintigraphy Imaging of radio-labelled microspheres in rabbits was done using two groups each consisting three animals. The SPECT CT imaging clearly depicted the retention of the formulation in the stomach and upper part of intestine thereby releasing the drug materials from there. A The scintigram represents the initial position of the radiolabelled formulation at time $0 \mathrm{~h}$. From the image, the location of the dosage form can be clearly seen and almost negligible amount of drug is released at the given time. B The scintigram obtained after time $1 \mathrm{~h}$ clearly represents the location of the dosage form with the subsequent release of the drug from that specific area as indicated by the dark colour dots. $\mathbf{C}$ At time interval of $4 \mathrm{~h}$, the scintigram obtained shows the presence of the dosage form in the same location within the $\mathrm{Gl}$ tract with further drug release from there this justifies the mucoadhesive nature of the prepared microsphere formulation. D The scintigram obtained at time interval of $6 \mathrm{~h}$ gives the further indication of the location of the formulation which supports the design concept of this current study. It can be clearly seen that the drug is still adhered to the mucosal lining in the upper part of intestine with the active medicament slowly releasing from there
} 


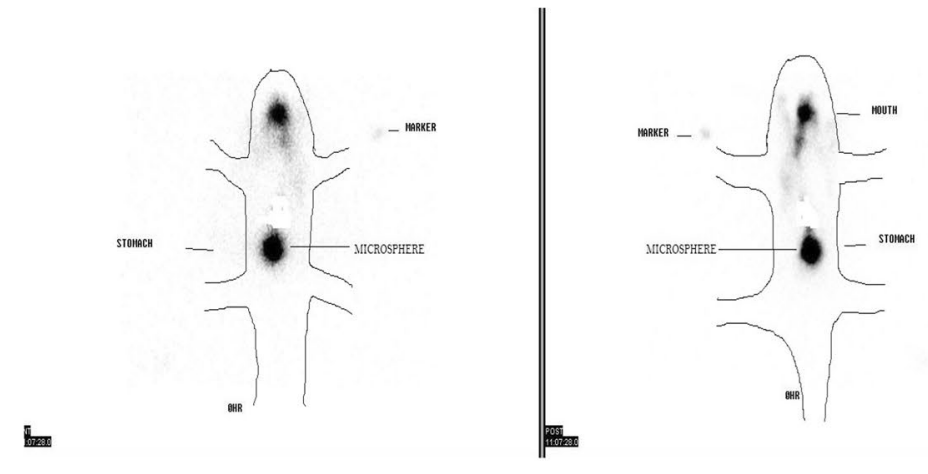

A. Gamma Scintigraphy image at time 0 hours after administration of the radiolabelled microsphere

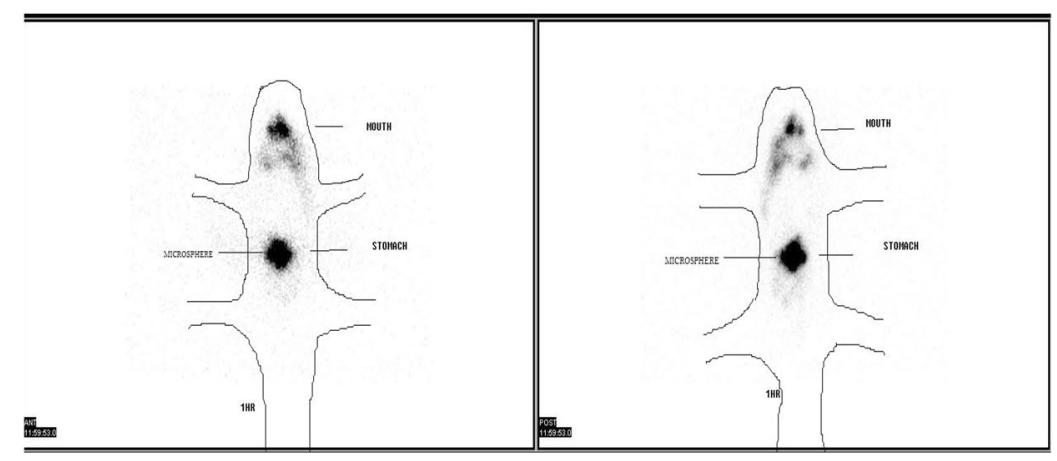

B. Gamma Scintigraphy image at time 1 hours after administration of the radiolabelled microsphere

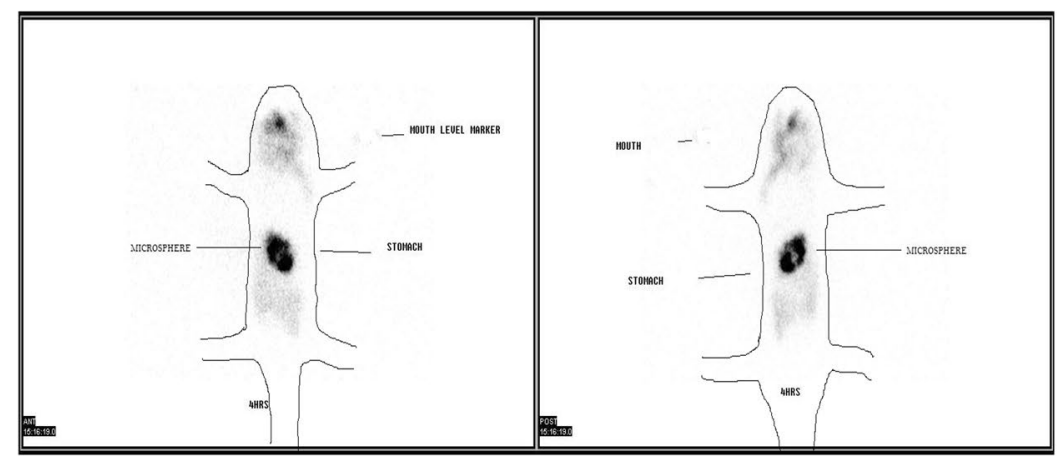

C. Gamma Scintigraphy image at time 4 hours after administration of the radiolabelled microsphere

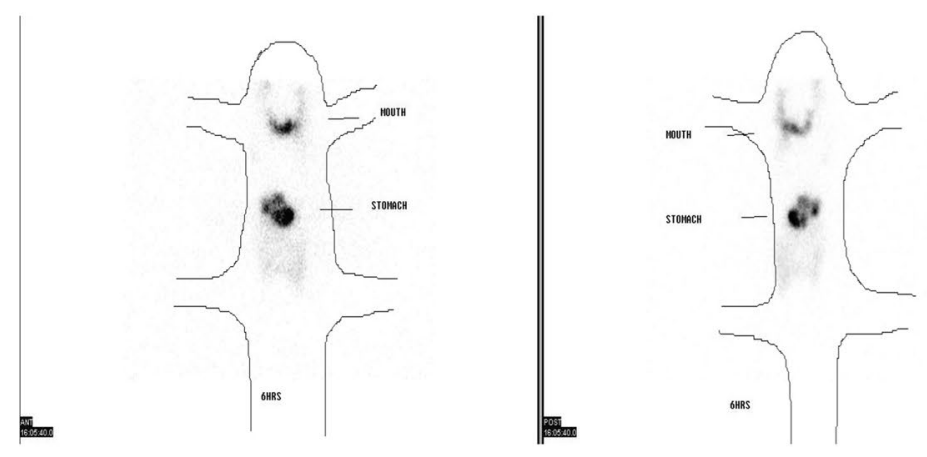

D. Gamma Scintigraphy image at time 6 hours after administration of the radiolabelled microsphere

Fig. 7 (See legend on previous page.) 


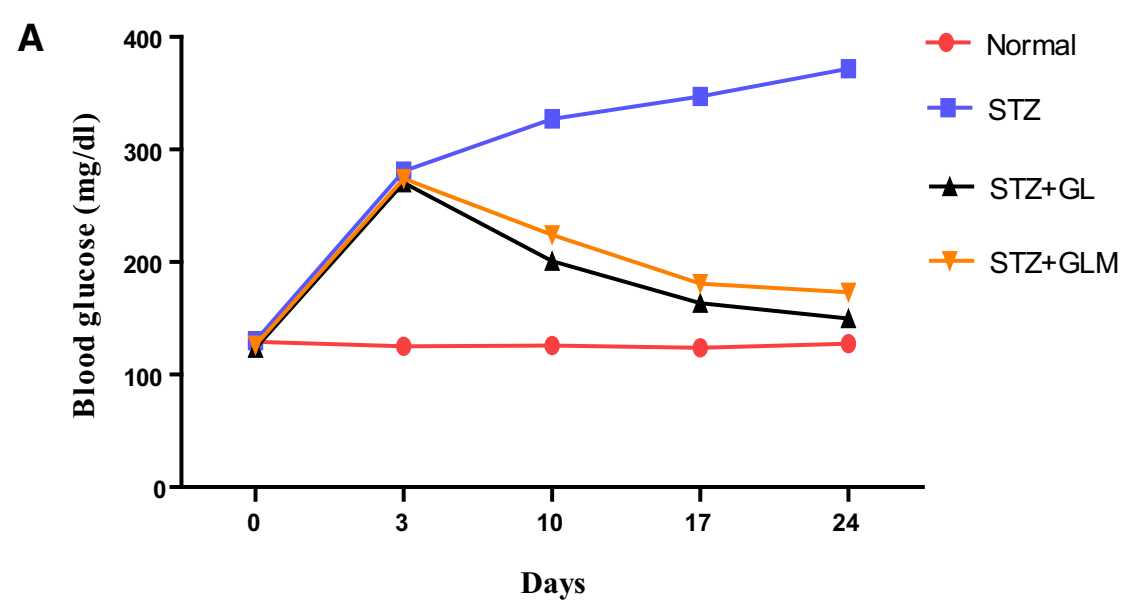

\section{B}
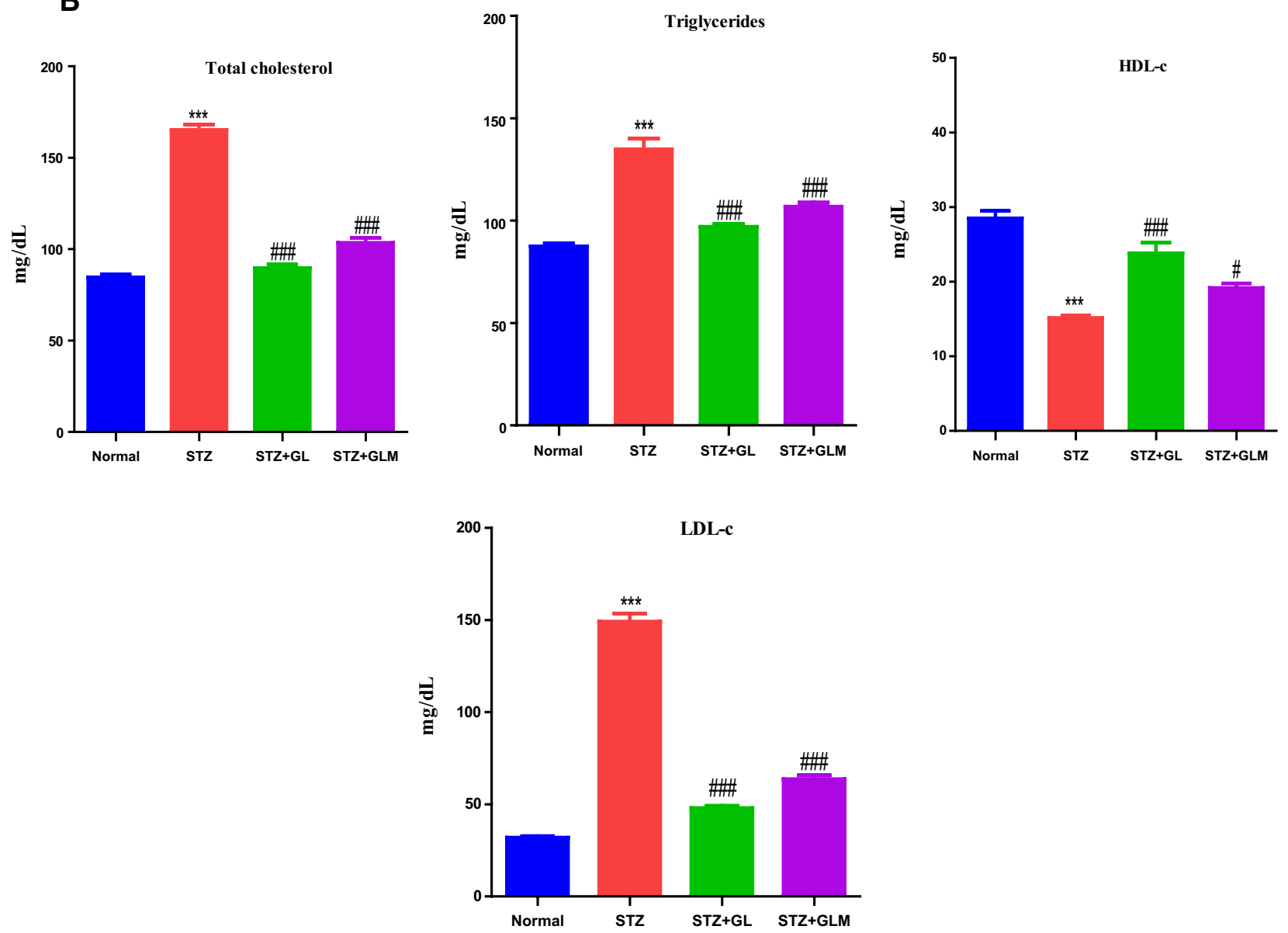

Fig. 8 The STZ diabetic model used for estimation of the effective nature of the prepared microsphere is resembled here. As seen in the red line, there was no such enhancement of serum blood glucose level when estimated at different time interval of 0 days, 3 days, 10 days, 17 days and 24 days. The blue line indicates the gradual increase in serum blood glucose level at different time interval for the STZ treated group (A). Similarly, the black line and the yellow line represent the effectiveness of the standard drug glibenclamide and the prepared MSB-Starch microsphere loaded with gliclazide, respectively, in controlling the serum blood glucose level when estimated in the given time interval. The effect of the microsphere on the lipid profile like total cholesterol, triglycerides, HDL-C and LDL-c is represented here. There was a subsequent increase in the total cholesterol, triglycerides, LDL-c level with the overall decrease in the HDL-c level as seen in the STZ treated group represented by the red block (B). There was no such alteration in the above parameters in the normal group as seen in the blue block. The green and violet block represents the effectiveness of the standard drug glibenclamide and the prepared microsphere in the management of the lipid profile 


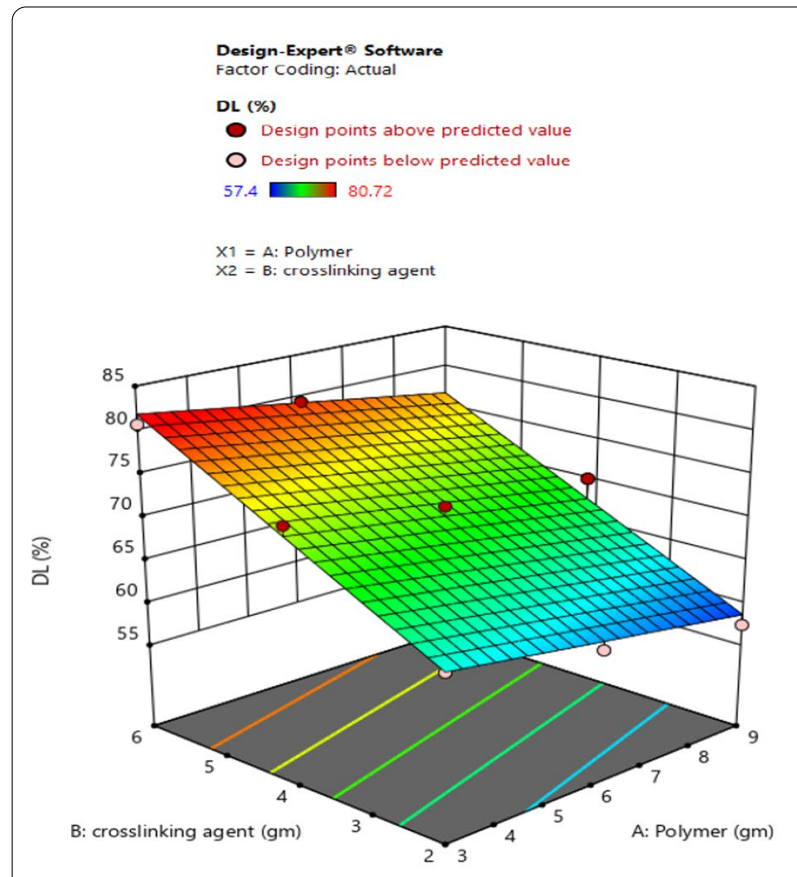

9A

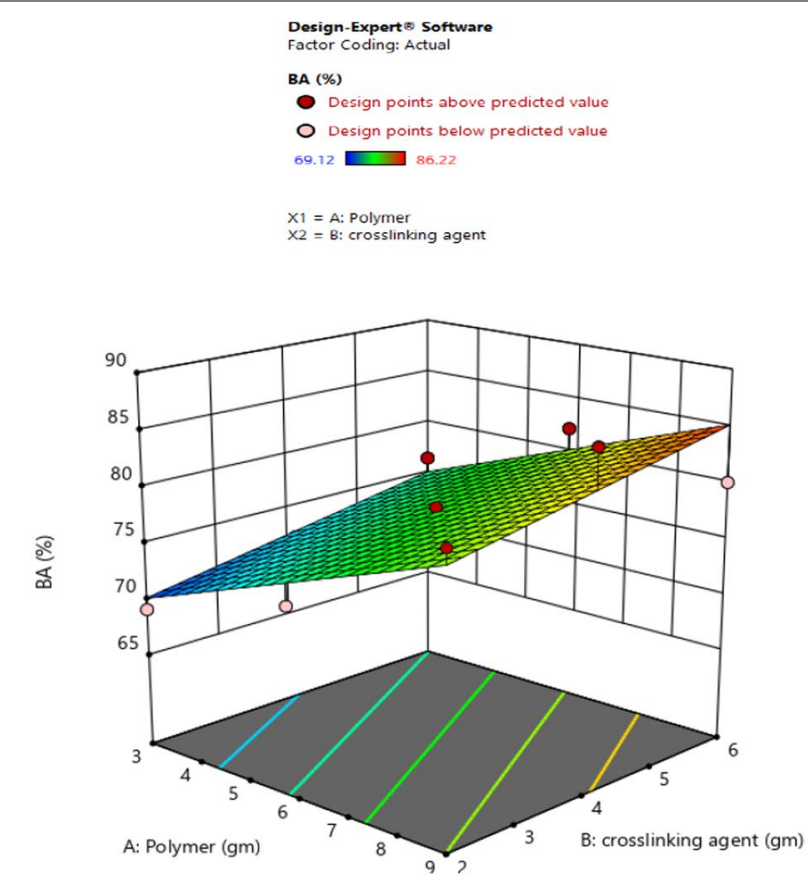

9B
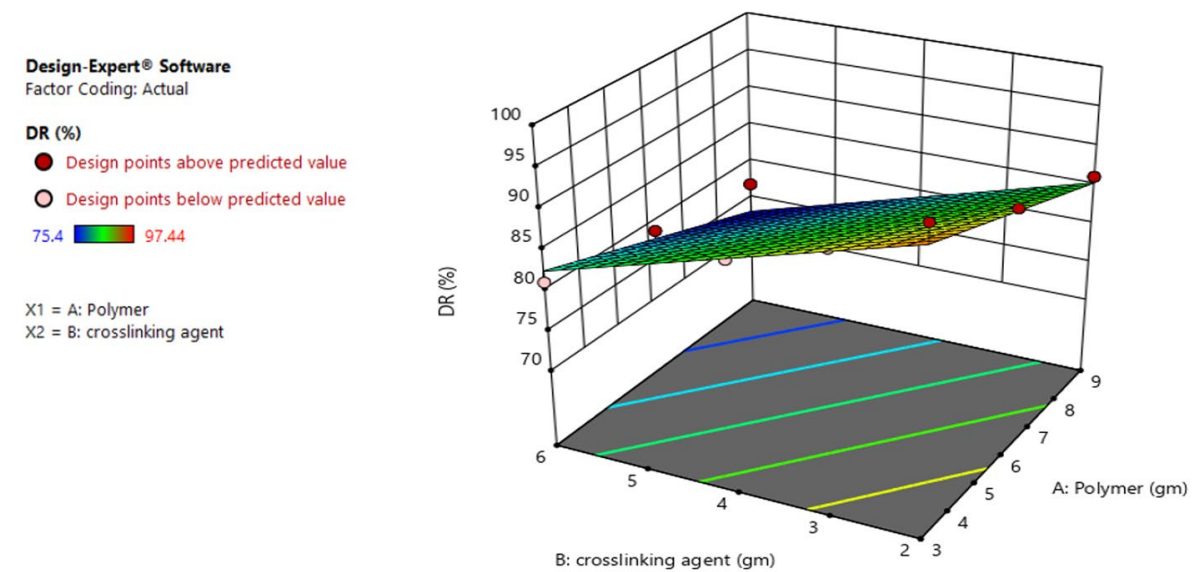

9C

Fig. 9 The 3D plot generated from the design expert software a 3D plot for \% drug loading b 3D plot for \% bio adhesion c 3D plot for \% drug release

with $\mathrm{Na}+$ ions present in the buffer solution where phosphate ions behave like sequestering agent for calcium. This leads to the initial disintegration of MSB-alginate, thereby causing erosion from the matrix and finally leading to the dissolution of the swollen microspheres.

The study data showed that the MSB-Starch alginate microsphere loaded with gliclazide might swell up initially in the stomach and then following its relocation in the upper part of the intestine where gliclazide will get absorbed. Finally, these microspheres will swell more and thus will release the loaded drug gliclazide.

The assessment of mucoadhesion provides an idea of the strength of association of the formulation with the mucosal surface. On performing the in vitro wash-off test, a speedy nature of wash-off was seen in intestinal $\mathrm{pH}$ compared to the acidic one shown in Fig. 4A, B. This reduction in mucoadhesion in the intestinal $\mathrm{pH}$ can be assumed because of the degradation of $\mathrm{Ca}++$ ions 


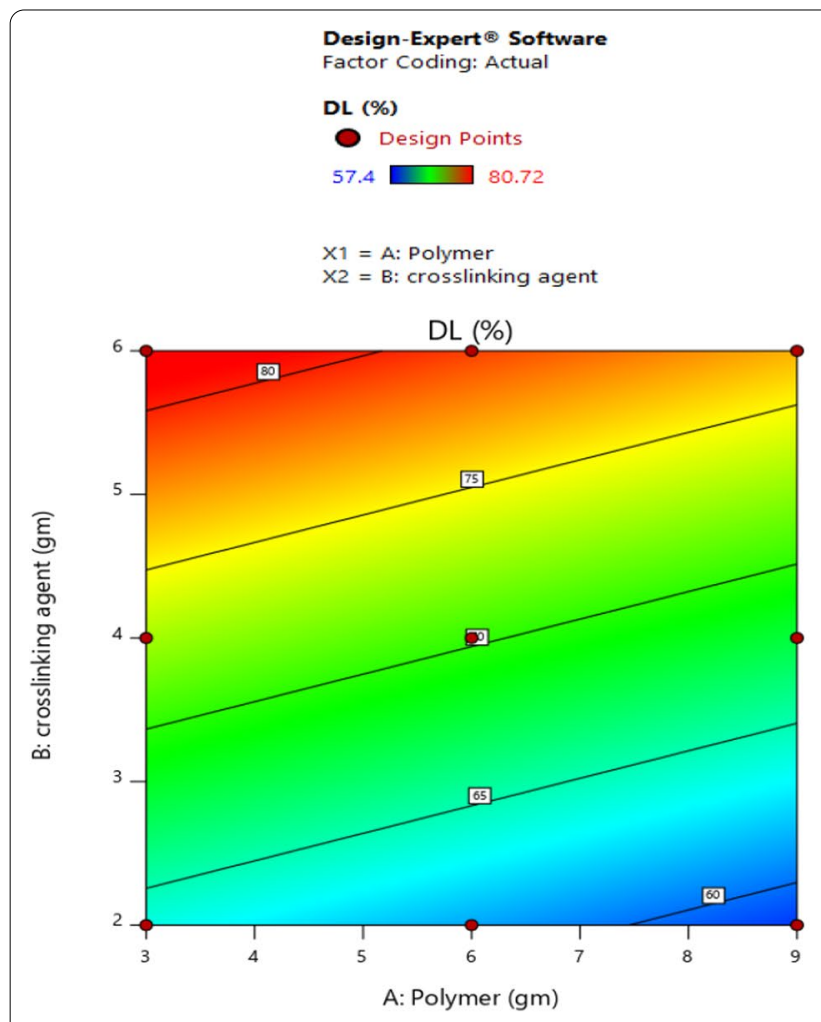

$10 \mathrm{~A}$

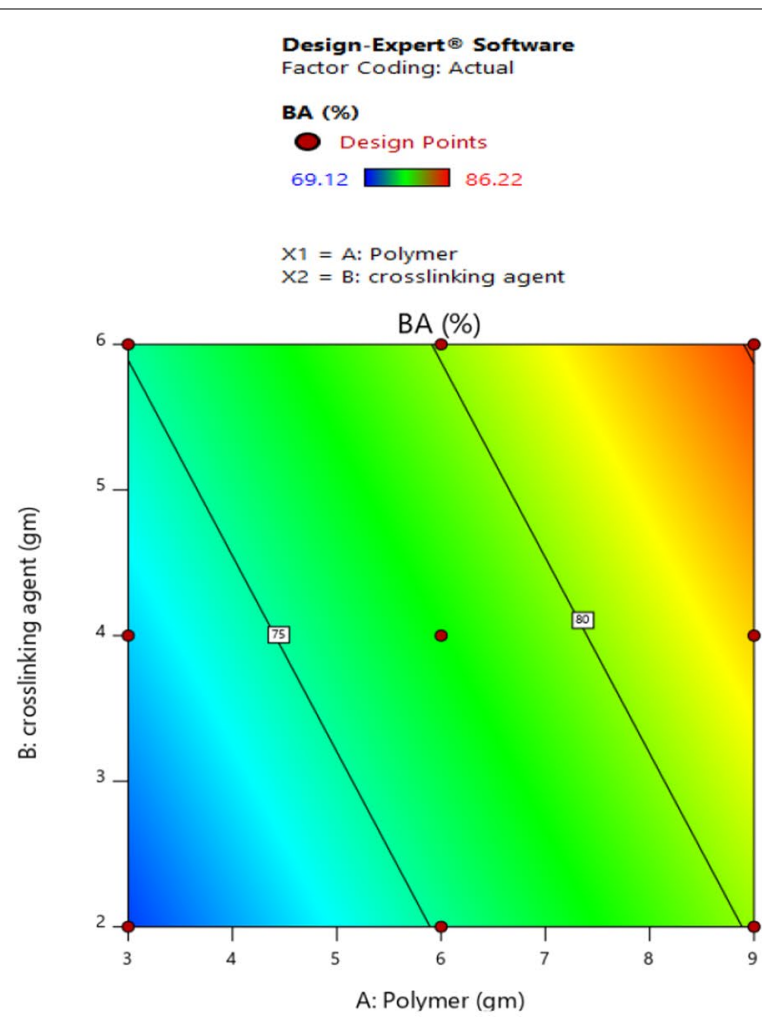

10B

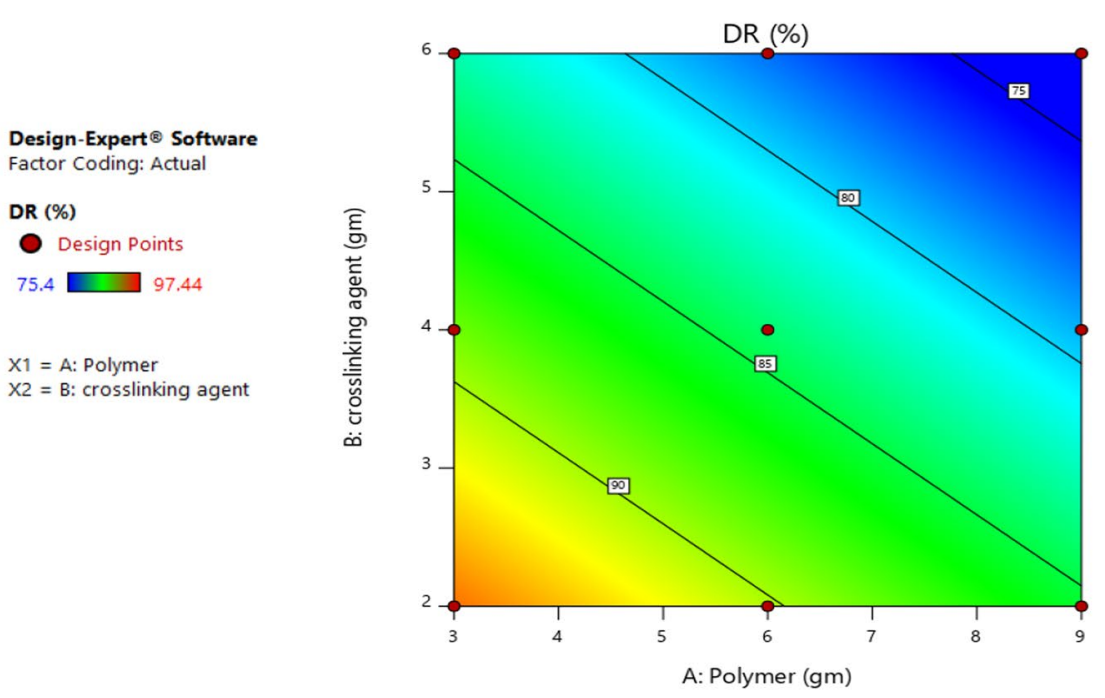

$10 \mathrm{C}$

Fig. 10 The contour plots generated by the software to evaluate the relationship between polymer concentration and amount of cross-linker and their effect on different parameters $\mathbf{A}$ contour plot for \% drug loading B contour plot for $\%$ bio adhesion c contour plot for \% drug release

and also could be because of $\mathrm{COOH}$ groups' ionization with other functional groups to enhance the aqueous solubility of the prepared mucoadhesive microsphere, thereby decreasing the mucoadhesiveness. The polymers, being hydrophilic, tend to absorb water, thereby swelling up leading to mucoadhesion with the mucosal membrane layer. Moreover, certain functional groups like hydroxyl and carboxyl present in the starch can also form 


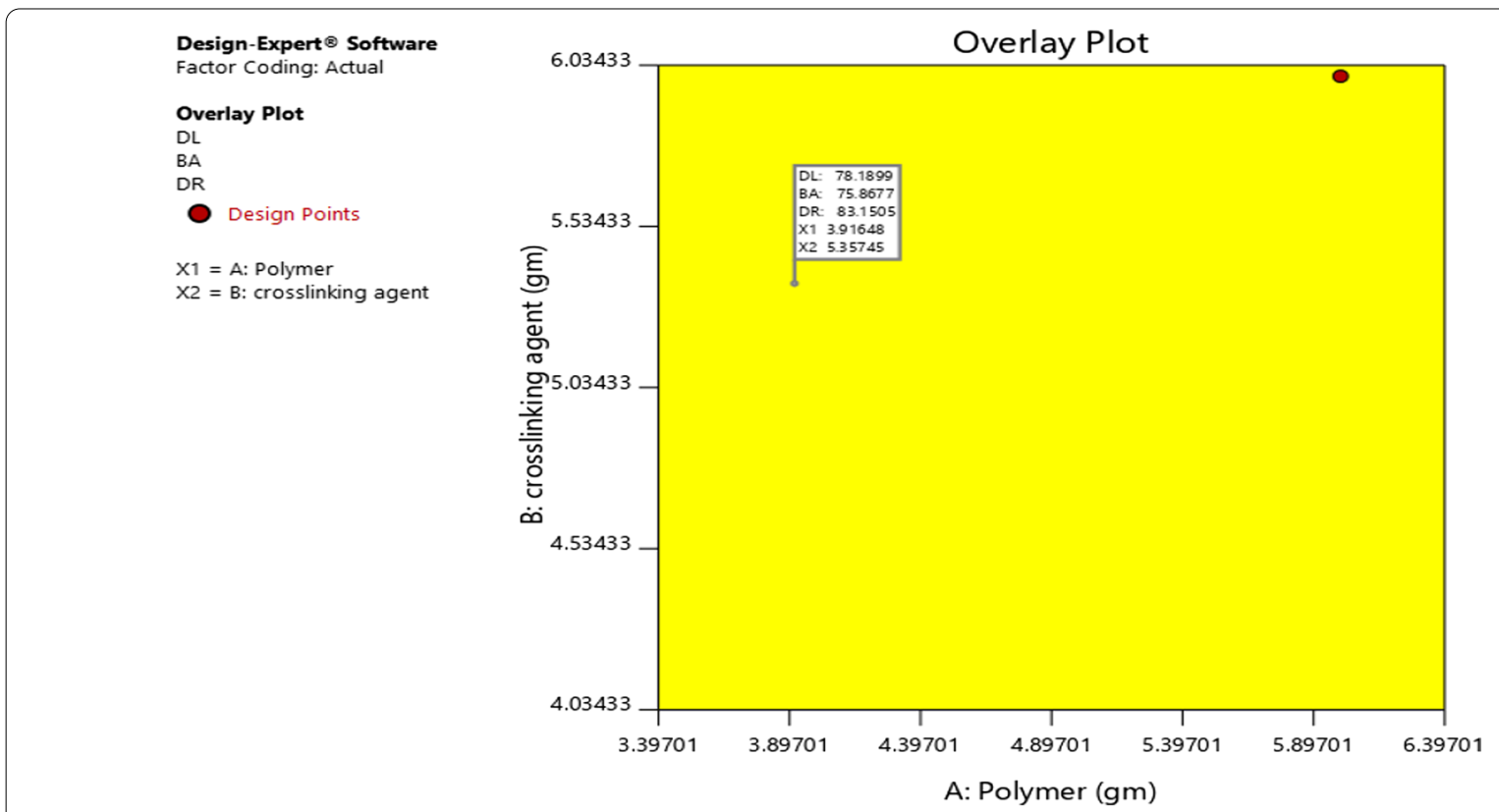

Fig. 11 The overlay plot of the desirability function generated by the software that provided the details of the optimized batch giving the desired optimum results required in our study purpose

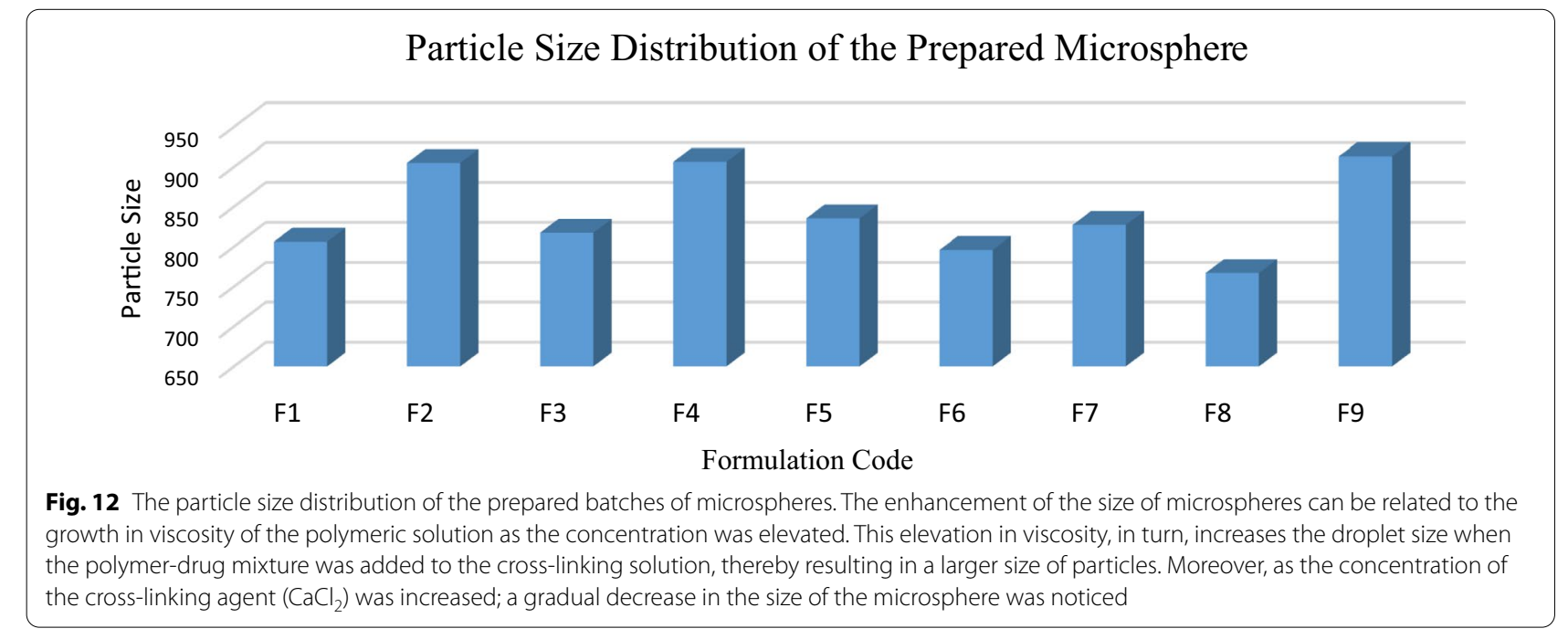

hydrogen bonds with the mucin molecules present in the intestinal tissues [44]. The results from this in vitro study indicate the mucoadhesiveness property of the prepared microsphere.

As seen in the FTIR spectra of gliclazide Fig. 1A, there was presence of a number of prominent and definite peaks indicating the presence of certain functional groups. The peaks seen at $1712.67 \mathrm{~cm}^{-1}$ and $1434.94 \mathrm{~cm}^{-1}$ were mostly because of the $\mathrm{C}=\mathrm{C}$ stretching occurring inside the benzyl ring of gliclazide structure. The presence of $\mathrm{NH}$ groups was confirmed by the peaks at $3186.86,3110.81,2933.35,2865.86$, $2834.51 \mathrm{~cm}^{-1}$ wave numbers. At $1345.81 \mathrm{~cm}^{-1}$, the stretching is mostly seen because of $\mathrm{S}=\mathrm{O}$ asymmetrical groups. The occurrence of $\mathrm{S}=\mathrm{O}$ symmetrical vibrations was seen from the prominent peak observed at $1160 \mathrm{~cm}^{-1}$. Furthermore, there was occurrence of $\mathrm{C}-\mathrm{O}$ stretching as seen in $1085.08 \mathrm{~cm}^{-1}$, an aromatic $P$ substitution phenyl at $916.63 \mathrm{~cm}^{-1}$ the presence of 
aromatic ring at $664.80 \mathrm{~cm}^{-1}$ and the occurrence of $\mathrm{C}=\mathrm{C}$ bending as seen by the peak at $994.10 \mathrm{~cm}^{-1}$.

In the FTIR spectra of sodium alginate Fig. 1b, the appearance of bands at 1590.13 and $1406.84 \mathrm{~cm}^{-1}$ resembles the peak appearing because of the symmetric carboxylate salt groups. Similarly, the peak obtained at around $1295.13 \mathrm{~cm}^{-1}$ is due to $\mathrm{C}-\mathrm{C}$ stretching, the peak at $1080.70 \mathrm{~cm}^{-1}$ is because of $\mathrm{C}-\mathrm{O}$ stretching and the peak obtained at $1025.11 \mathrm{~cm}^{-1}$ is due to $\mathrm{C}-\mathrm{O}-\mathrm{C}$ stretching. Also, the peak seen at $944.55 \mathrm{~cm}^{-1}$ is due to $\mathrm{C}-\mathrm{O}$ stretching attributing to the saccharide structure. The FTIR spectra of the starch obtained from Musa balbisiana species Fig. 1C showed characteristic peak at $3251.22 \mathrm{~cm}^{-1}$ which can be attributed due to $\mathrm{O}-\mathrm{H}$ stretching, the peak at $2921.49 \mathrm{~cm}^{-1}$ is because of $\mathrm{C}-\mathrm{H}$ stretching, $1630 \mathrm{~cm}^{-1}$ is due to $\mathrm{C}-\mathrm{O}$ that is associated with the $\mathrm{OH}$ group. There was the occurrence of a prominent peak at $1334.76 \mathrm{~cm}^{-1}$ that is attributed because of $\mathrm{C}-\mathrm{H}$ symmetric bending. The peak obtained at $1148.55 \mathrm{~cm}^{-1}$ is likely to be because of $\mathrm{C}-\mathrm{O}-\mathrm{C}$ asymmetric stretching, and the one obtained at $1075.22 \mathrm{~cm}^{-1}$ is because of $\mathrm{C}-\mathrm{O}$ stretching. There was appearance of peaks at $997.35 \mathrm{~cm}^{-1}$ and $857.36 \mathrm{~cm}^{-1}$ because of the $\mathrm{C}-\mathrm{O}-\mathrm{C}$ ring vibration in carbohydrates. There was also the occurrence of characteristic peaks of the drug in the FTIR spectrum of formulation Fig. 1d that was prepared by using both the polymer combination.

The DSC thermogram of pure GLZ powder Fig. 2a exhibited a sharp endothermic peak at $175.80{ }^{\circ} \mathrm{C}$ which corresponds to its melting point. The onset of reaction started at $168.64{ }^{\circ} \mathrm{C}$ and end set at about $180^{\circ} \mathrm{C}$ with glass transition lag around $6.65^{\circ} \mathrm{C}$. On the other hand, the DSC thermogram of alginate Fig. $2 \mathrm{~B}$ exhibited an endothermic peak (dehydration peak) at $111.7^{\circ} \mathrm{C}$ and also exhibited an exothermic peak (decomposition peak) at $248^{\circ} \mathrm{C}$. There was no change observed in the melting point as well as in the glass transition lag. However, there were special peaks resembling the melting point of sodium alginate at $111.7^{\circ} \mathrm{C}$, starch at $117.84^{\circ} \mathrm{C}$ Fig. $2 \mathrm{C}$. It was also observed that there was an influence of the excipients in altering the intensity of melting point peak of GLZ by absorbing heat and this has not occurred because of any interactions. The interpretational data found above can clearly indicate that the drug crystalline nature remains intact and as there is no any interaction, it does not undergo polymorphism.

The DSC thermogram of the drug and excipient mixtures Fig. 2D along with the FTIR spectrum of the drug and excipient mixture Fig. 1E clearly shows that there is no any interaction between the drugs and the excipients used in the preparation of microsphere.
The IST study incorporates the storage of drug and the excipients with or without the presence of moisture at an elevated temperature. These conditions are basically used to accelerate the process of drug ageing and interactions with the available excipients. In conducting this study, $5 \mathrm{mg}$ of drug was accurately weighed out into vials with addition of equal quantities of excipients. No characteristic changes in colour were seen when physical evaluation of the samples was carried out at different time intervals. Furthermore, the UV spectroscopic method was also employed for estimating the percentage purity of the pure drug along with the sample containing the mixture of drug and polymer after 3 weeks and the assay results was found to be within the acceptable limit (Table 3) that clearly indicates the stable behaviour of GLZ when used with polymer starch and sodium alginate. Also peak matching technique carried out by spectral analysis exhibited the presence of specific functional groups for the pure drug and the mixture of drug and excipients which omits out the possibilities of any molecular changes in the drug molecule brought by the excipients. Finally, the availability of any unreacted excipients expressing the similar functional group with the parent drug was nullified by estimating the wavelength maxima $(\lambda \max )$ of the samples by using UV spectroscopy. There was no change in the Drug wavelength maxima which relates to the compatibility between the parent drug and its physical mixtures used (Table 3).

Following optimization, the equation obtained for drug release study provided clear evidence that the term X1, i.e. the Polymer concentration had an antagonistic effect as seen by the negative sign in the coefficient X1, whereas term X2 had a synergistic effect as indicated by the positive sign in the coefficient $\mathrm{X} 2$. It can be related that as the polymer concentration increases, the drug loading decreases. The highest drug entrapment efficiency was seen as the polymer ratio was kept towards the lower side, and the concentration of the cross-linking agent was kept towards the higher side. As observed in 3D plot of drug loading Fig. 9A, an upper level was observed for low polymer concentration and higher cross-linking and gradually decreases with the increase in concentration of polymer and lowering the amount of cross-linker. The contour plots Fig. 10A were also built to evaluate the relationship between polymer concentration and amount of cross-linker and their effect on drug loading. In the equation for bioadhesiveness study, it is clear that both the coded term X1, i.e. the Polymer concentration and X2 the concentration of cross-linking agent has a synergistic effect as seen by the positive sign on both the factors in the equation. The positive sign in the coefficient X1 suggested that as the concentration of polymer (MSB starch: Alginate) increases, the bioadhesiveness of 
the microsphere increases. The polymers, being hydrophilic, tend to absorb water, thereby swelling up leading to mucoadhesion with the mucosal membrane layer. As observed in the figure for 3D plot Fig. 9B of bioadhesion, it was seen that the bioadhesion was at upper level when the polymer concentration and concentration of crosslinker was gradually increased and with the decrease in concentration of both, the bioadhesion was found to be lowered. The contour plots Fig. 10B were also built to evaluate the relationship between polymer concentration and amount of cross-linker and their effect on bioadhesion.

The negative sign in both the coded term X1 and X2 for the equation obtained for drug release study indicated the antagonistic effect. Coefficient X1 indicated that as the concentration of polymer increases, the drug release tends to decrease and this can be attributed to an increase the density of the polymer matrix and also in the diffusion path length that the drug molecules have to traverse. The amount of cross-linking agent had also an impact in the rate of drug release. It was seen that the rate and extent of drug release was significantly decreased with an increase in concentration of the cross-linking agent as depicted by the negative sign of the coefficient X2 in the equation. This was probably because of the formation of tight junction between the MM/GG residues of sodium alginate with calcium ions.

For 3D plot Fig. 9C of drug release, it was seen that the drug release was at upper level when the polymer and cross-linker concentration was gradually decreased and with the increase in concentration of both, the drug release was found to be lowered. The contour plots Fig. $10 \mathrm{C}$ were also built to evaluate the relationship between polymer concentration and amount of crosslinker and their effect on Drug Release. The predicted residual sum of squares (PRESS) that is also known as the sum of squared residuals (SSR) or also mostly known as sum of squared errors of prediction (SSE). This PRESS value provides an indication of how well a model fits the data provided and it is always recommended that the PRESS value should always be relatively small for a chosen model in comparison with the other models that is under consideration [45]. The fitness of data is mostly expressed by those polynomial models showing a lower PRESS value, and such model is mostly selected. In the current experiment, the PRESS values were calculated for the linear, 2FI, quadratic and cubic models for both the independent variables (X1 and X2). Results indicated the suitability of the linear model as compared other models with smaller PRESS values for both $\mathrm{X} 1$ and $\mathrm{X} 2$. The summary of model statistics for the responses is shown in Table $2 b$.
As seen in the FESEM analysis, there was exhibition of smooth surface with occasional wrinkles and the presence of polymeric debris. The formation of wrinkles and debris may be associated with the method of preparation as by simultaneous gel microsphere preparation and because of the formation of polymer blend matrix. This can also be thought because of the partial collapsing of the polymeric gel network during the process of drying. The occurrence of drug crystals on the surface can be resembled because of the migration of the drugs along with water to the surface in the drying process [46].

The prepared GLZ-loaded MSB Starch-alginate microsphere exhibited a prolonged release of the drug over a period of $12 \mathrm{~h}$. The rate of drug release from the microsphere in PBS of pH 7.2 was contributed basically because of the rapid swelling followed by the erosion of the beads. There are reports that the swelling of the alginate beads in the presence of $\mathrm{Ca} 2+$ capturing agents is dependent basically on the continuous displacement of $\mathrm{Ca} 2+$ ions within the beads [47]. Furthermore, the existence of phosphate ions which mostly acts as calcium sequestering agent can also enhance the overall swelling [43]. With the increase in alginate concentration, the rate of release of gliclazide from the prepared beads was found to decrease. The overall reduction in this release rate can be described with the enhancement in the magnitude of swelling and also the layer of gel thickness which mostly acts as the major obstacle in permeation of the dissolution fluid which in turn retards the drug diffusion from the swollen alginate matrix. Also, there is an increment in the density of the beads with the increase in concentration of polymer and this suggests that the beads developed at high concentration of polymers are mostly compact in nature and are having a less porous nature when compared with the beads developed using low polymer content [48].

Also, the correlation coefficients values for zero order (R0), First-order (R1) and Higuchi (RH) kinetics for all the prepared formulations were determined. On observation, it was found that the drug release from prepared microsphere follows zero-order kinetics $(\mathrm{R} 2=0.956-$ 0.994) over the time period of $12 \mathrm{~h}$. and this mostly indicates the nature of controlled drug release from these formulations. Furthermore, in order to differentiate the two competing release mechanisms, i.e. Fickian diffusional release $(n \leq 0.5)$ and case II transport $(n \geq 1)$, the Korsmeyer-Peppas model was also employed. It was seen that the diffusion coefficient (n) ranged from 1.181 to 1.237 , which gives a clear indication of the drug release following the super case II transport mechanism that is basically controlled with the swelling followed by the relaxation of the polymer matrix [49]. 
The prime goal of utilization of formulation optimization in pharmaceuticals is mostly to find out the various levels of variable which generally affects the responses chosen and also to estimate out the different level of variables which in turn will help in the formulation of a product of robust nature having attributed characteristics of quality. In the optimization process, several response parameters which can affect the overall quality of the product were basically considered using the minimum amount of excipients. On analysis, an overlay plot Fig. 11 of the desirability function provided the details of the optimized batch giving the desired optimum results required in our study purpose. On comparison with our prepared batches, the F8 batch closely relates to the optimized batch and this can be considered as the optimized batch [35-38].

After the oral administration of the optimized lyophilized batch of mucoadhesive microsphere (F8), the estimation of essential pharmacokinetic parameters like $C_{\max }$, $T_{\max }, \mathrm{Auc}_{0-\mathrm{t}}$ and $\mathrm{AUC}_{0 \text {-infinity }}$ was carried out (Table 6). The maximum concentration $\left(C_{\max }\right)$ achieved after oral administration of GLZ powder was $2.013 \pm 0.035 \mu \mathrm{g} /$ $\mathrm{ml}$ compared to $14.407 \pm 0.300 \mu \mathrm{g} / \mathrm{ml}$ for the lyophilized GLZ-loaded mucoadhesive microsphere. There was about a 6.8 -fold increase in this maximum plasma drug concentration which mostly indicates a marked enhancement in the amount of drug absorption after the GLZ-loaded microsphere was administered orally. The statistical analysis data also showed a highly significant $(\mathrm{p} \leq 0.001)$ increase in the values of $C_{\max }$ following the administration of GLZ-loaded microsphere when compared with raw GLZ powder. The parameters AUC $0-\mathrm{t}$ and $\mathrm{AUC}_{0-\alpha}$ estimate the total drug exposure and are considered mostly for measuring the bioavailability. The GLZ-loaded microsphere showed $\mathrm{AUC}_{0-\alpha}=127 \mu \mathrm{g} \mathrm{h} / \mathrm{ml}$, while the raw GLZ had this value $=47 \mu \mathrm{g} \mathrm{h} / \mathrm{ml}$ providing a 2.7-fold enhancement in drug bioavailability which is highly significant. This increase in bioavailability gave an indication about the higher gastro-intestinal uptake of

Table 6 Pharmacokinetic parameters of the optimized formula (F8) an draw GLZ powder after oral administration to rats in a dose corresponding to $10 \mathrm{mg} / \mathrm{kg} \mathrm{GLZ}$

\begin{tabular}{lccccc}
\hline Pk parameters & \multicolumn{2}{l}{ Gliclazide API } & & \multicolumn{2}{l}{$\begin{array}{l}\text { Gliclazide } \\
\text { microspheres }\end{array}$} \\
\cline { 2 - 3 } & Mean & SD & & Mean & SD \\
\hline$C_{\text {max }}(\mu \mathrm{g} / \mathrm{ml})$ & 2.013 & 0.035 & & 14.407 & 0.300 \\
$T_{\max }(\mathrm{h})$ & 4.000 & 0.000 & & 4.000 & 0.000 \\
AUC 0_24 $\left(\mu \mathrm{g}^{*} \mathrm{~h} / \mathrm{ml}\right)$ & 26.108 & 0.217 & & 105.870 & 0.731 \\
AUC t_infi $\left(\mu \mathrm{g}^{*} \mathrm{~h} / \mathrm{ml}\right)$ & 47.586 & 1.482 & & 127.516 & 0.857 \\
\hline
\end{tabular}

Data are expressed as mean \pm SD the GLZ microsphere as compared to raw GLZ powder. The time to reach maximum plasma concentration $\left(T_{\max }\right)$ is a measure of the rate of drug absorption. The raw GLZ powder shows an erratic absorption with a higher intersubject variation with the $T_{\max }$ ranging from as low as half an hour up to $4 \mathrm{~h}$. This type of behaviour has already been reported and can be related to its early dissolution in the stomach leading to more variability in the absorption in the intestine [50].

Furthermore, there was a bimodal release seen in case of raw GLZ with 2 peaks one at $1 \mathrm{~h}$ and the other occurring at $4 \mathrm{~h}$ that was already reported by Davis et Al. [51] signifying that GLZ underwent enterohepatic circulation together with its absorption from a second more distal site in the GIT. The statistical analysis data were used in comparing $T_{\max }$ of both GLZ powder and GLZ microsphere. GLZ-loaded microsphere exhibited a prolong release with $T_{\max }=4 \mathrm{~h}$ when compared to $T_{\max }$ of $1 \mathrm{~h}$ in GLZ powder. Also, there was a decrease in the apparent total clearance of GLZ microsphere when compared with the pure drug confirming its slow release pattern thereby prolonging its release.

From the gamma scintigraphy images, it can be clearly understood that the dosage form was retained in the stomach for more than $4 \mathrm{~h}$, and the drug was released over time in the intestinal region. The images obtained for both test and standard were compared, and it was seen that the test formulation showed a greater degree of bioadhesiveness, and the medicament was gradually released from there in a sustained manner. However, in the case of the standard, burst liberation was seen, thereby omitting any evidence of mucoadhesion. The study data also indicated that about $80 \%$ of the medicament was released in $2 \mathrm{~h}$ in the standard formulation; however, in the case of test formulation, it took more than $6 \mathrm{~h}$ to release the total drug.

Finally, the antidiabetic study was carried out using the STZ-induced diabetic rat model. The optimized formulation (F8) of GLZ-loaded MSB starch microsphere was administered orally. Previous study reports for antidiabetic activity by using pure gliclazide reveals that, there was a rapid reduction in blood glucose level that was observed for $2 \mathrm{~h}$ and after that the blood sugar level was recovered towards the normal. [52] However, there was a reduction in blood glucose level that reached a maximum within 3-4 $\mathrm{h}$ and was sustained over $12 \mathrm{~h}$ following the administration of the GLZ-loaded microsphere. A reduction of $25 \%$ in blood glucose level is considered a significant hypoglycemic effect [53-57]. Hence, the sustained hypoglycemic effect of MSB starch GLZ-loaded microsphere was observed for a longer period of time. The above study suggested that the Micropshere containing gliclazide undergoes swelling slowly in the stomach 
thereby adhering accordingly to the stomach mucosa. This allows more amount of gliclazide to get absorbed which minimizes the diffusion barriers to enhance the absorption period by prolongation of the gastric residence time of the drug. The microspheres than moved subsequently towards the upper part of the intestine where they underwent more swelling and released the drug through the polymeric gel layer that was developed at the matrices periphery. The study results clearly demonstrated the potential of the GLZ-loaded MSB starch microspheres to enhance, prolong and also to control the gastrointestinal absorption of gliclazide.

\section{Conclusion}

MSB-Starch alginate microspheres loaded with gliclazide were formulated by the ionic gelation technique and were subjected to subsequent evaluation. The isolated starch was initially imparted with some physical and chemical modifications. The parameters like percentage yield, drug entrapment efficiency, size of the microsphere were within acceptable limits. Further studies suggested that the prepared microsphere possesses good mucoadhesiveness. Also, it was seen that the mucoadhesive microsphere showed a gradual swelling behaviour in the stomach and sticks to the stomach mucosal lining. It was further seen that this enhancement of residence in the stomach allowed more drugs to get absorbed by sufficient reduction in the diffusion barriers. Further swelling and drug release were seen when the microsphere reaches the upper part of the intestine. A polymeric gel layer formed at the matrix periphery causes the enhancement of drug release. The in vivo gastro-retention of the optimized formulation in rabbits using the technique of gamma scintigraphy justified the design concept and related the potential of this developed system for stomach-specific targeting. Moreover, it can be clearly understood that the prepared gliclazide-loaded MSB-Starch mucoadhesive microsphere has a profound effect in the management of diabetes. Hence, conclusion can be drawn that this Musa balbisiana starch is a potential mucoadhesive agent available from a natural source and can be utilized to formulate site-specific controlled release mucoadhesive microsphere orally, thereby enhancing the bioavailability of drugs.

\footnotetext{
Abbreviations

STZ: Streptozotocin; DEE: Drug entrapment efficiency; PBS: Phosphate buffer solution; USP: United States pharmacopoeia; Gl: Gastrointestinal; MBq: Megabecquerel; KeV: Kilo electron volts; NC: Normal control; GL: Glibenclamide; GLM: Gliclazide-loaded microsphere; LDL-c: Low-density lipoprotein cholesterol; HDL-c: High-density lipoprotein cholesterol.
}

\section{Supplementary Information}

The online version contains supplementary material available at https://doi. org/10.1186/s43094-021-00376-3.

Additional file 1. Arrive Checklist.

\section{Acknowledgements}

The authors wish to thank Assam Science and Technology University (ASTU), Guwahati, Assam, India for their continuous support and suggestion in carrying out the work.

\section{Authors' contributions}

ABA carried out designing of the current study. RB carried out collection, formulation and evaluation of the dosage form along with the animal study. $\mathrm{RB}$ drafted the manuscript and participated in the design and coordination of manuscript. $A B A$ and $K B$ reviewed the manuscript. All authors read and approved the final manuscript.

Funding

Not applicable.

Availability of data and materials

All data and materials are available upon request.

\section{Declarations}

Ethics approval and consent to participate

The experimental animal protocol approved by the Animal Ethical Committee of NETES Institute of Pharmaceutical Science (NIPS), Kamrup, Assam, India and care of animal was taken as per the guidelines of CPCSEA, Department of Animal Welfare, Government of India (Approval Number NIPS/AH/20/19).

\section{Consent for publication}

Not applicable.

\section{Competing interests}

The authors declare that they have no competing interests.

\section{Author details}

${ }^{1}$ Assam Science and Technology University (ASTU), Tetelia Road, Jalukbari, Assam 781013, India. ${ }^{2}$ NETES Institute of Pharmaceutical Science, NIPS Mirza, Santipur Kamrup, Assam 781125, India. ${ }^{3}$ Girijananda Choudhury Institute of Pharmaceutical Science, Tezpur, Assam 784501, India.

Received: 3 August 2021 Accepted: 29 October 2021

Published online: 24 November 2021

\section{References}

1. Prajapati ST, Patel LD, Patel DM (2008) Gastric floating matrix tablets: design and optimization using combination of polymers. Acta Phrmaceutica 58:221-229

2. MishraPK BajpaiM (2014) Formulation and evaluation of controlledrelease of telmisartan microspheres: in vitro/in vivo study. J Food Drug Anal 22:542-548

3. Roy SK, Prabhakar B (2010) Bioadhesive polymeric platforms for transmucosal drug delivery systems-a review. Trop J Pharm Res 9:91-104

4. Callens C, Adriaens E, Dierckens JP, Remon J (2001) Control Release 76:81-91

5. Alam MT, Parvez N, Sharma PK (2014) FDA-approved natural polymers for fast dissolving tablets. J Pharm 2014:1-6

6. Kulkarni V, Butte K, Rathod S (2012) Natural polymers - a comprehensive review. Int J Res Pharm Biomed Sci 3:1597-1613

7. Navarro DM, Abelilla JJ, Stein HH (2019) Structures and characteristics of carbohydrates in diets fed to pigs: a review. J Anim Sci Biotechnol 10:1-17 
8. Ibrahim MS, Wassefy NA, Farahat DS (2017) Biocompatibility of dental biomaterials. In: Biomaterials for oral and dental tissue engineering. Elsevier

9. Belattmania Z, Kaidi S, Atouani S, Katif C, Bentiss F, Jama C, Reani A, Sabour B, Vasconcelos V (2020) Isolation and FTIR-ATR and 1H NMR characterization of alginates from the main alginophyte species of the atlantic coast of Morocco. Molecules 25:1-9

10. Reddy KV, Nagabhushanam MV (2017) Crosslinked alginate hydrogel beads for ezetimibe. Int J Pharm Pharm Sci 9

11. Nayak AK, Pal D, Santra K (2013) Plantago ovata F. mucilage-alginate mucoadhesive beads for controlled release of glibenclamide: development, optimization, and in vitro-in vivo evaluation. J Pharm 2013:1-11

12. Temesgen S, Rennert M, Tesfaye T, Nase M (2021) Review on spinning of biopolymer fibers from starch 1-24.

13. Takeda Y, Priess J (1993) Carbohyd Res 240:265-275

14. Mohamad Y, Abdullah N, Muhammad N, Matias HM (2018) Application of starch and starch-based products in food industry. J Sci Technol 10:144-174

15. Bhattacharya K, Mathew J (2021) Development and validation of stabilityindicating UPLC method for the determination of gliclazide and its impurities in pharmaceutical dosage forms. Fut J Pharm Sci 7:95

16. Odeniyi MA, Onu RN, Adetunji OA (2011) Evaluation of bioadhesive properties of natural and modified banana starches. East Central Afr J Pharm Sci 14:34-42

17. Surini S, Anggriani V, Anwar E (2009) Study of mucoadhesive microspheres based on pregelatinized cassava starch succinate as a new carrier for drug delivery. J Med Sci 9:249-256

18. Luis A, Bello P, Edith AA, Laura SH, Octavio PL (1999) Isolation and partial characterization of banana starches. J Agric Food Chem 47:854-857

19. SengelTurk CT, Hascicek C (2017) Design of lipid-polymer hybrid nanoparticles for therapy of BPH: part I. Formulation optimization using a design of experiment approach. J Drug Deliv Sci Technol 39:16-27

20. Ghumman SA, Bashir S, Noreen S, Khan AM, Riffat S, Abbas M (2018) Polymeric microspheres of okra mucilage and alginate for the controlled release of oxcarbazepine: in vitro \& in vivo evaluation. Int J Biol Macromol 111:1156-1165

21. Ahmed AB, Bordoloi R, Bhattacharya K (2022) Development and validation of a RP-UHPLC method for estimation of gliclazide loaded microsphere. Res J Pharm Tech 2:15

22. Nerkar PP, Gattani S (2011) In vivo, in vitro evaluation of linseed mucilage based buccal mucoadhesive microspheres of venlafaxine. Drug Delivery 18:111-121

23. Pani NR, Nath LK, Acharya S (2011) Compatibility studies of nateglinide with excipients in immediate release tablets. Acta Pharm 61:237-247

24. Bera K, Khanam J, Mohanraj KP, Mazumder B (2014) Design and evaluation of mucoadhesive beads of glipizide as a controlled release drug delivery system. J Microencapsul 31:220-229

25. Dorniani D, Zobir M, Shaari AH, Ahmad Z (2013) Preparation and characterization of 6 mercaptopurine-coated magnetite nano particles as a drug delivery system. Drug Des Dev Ther 7:1015-1026

26. Bansal G, Singh M, Jindal KC (2007) Forced degradation study on gliclazide and application of validated stability-indicating HPLC-UV method in stability testing of gliclazide tablets. Chromatographia 66:751-755

27. Sharma VK, Mazumder B (2014) Gastrointestinal transition and antidiabetic effect of Isabgol husk microparticles containing gliclazide. Int J Biol Macromol 66:15-25

28. Zhang Y, Huo M, Zhou J et al (2010) DD solver: an add-in program for modelling and comparison of drug dissolution profiles. AAPS J 12:263-271

29. Narasimhan B (2001) Mathematical models describing polymer dissolution: consequences for drug delivery. Adv Drug Deliv Rev 48:195-210

30. Khalil SS, Ali HA, Al-Saadawy HA, El-Dawy K (2018) Combination of gliclazide drug and lupin seeds powder alleviate hyperglycemia on induced-diabetic rats receiving high-fat high fructose/sucrose diet. Slov Vet Res 55:273-281

31. Burcelin R, Eddouks M, Maury J, Kande J, Assan R, Girard J (1995) Excessive glucose production, rather than insulin resistance, accounts for hyperglycemia in recent-onset streptozotocin-diabetic rats. Diabetologia 38:283-290

32. Kim JD, Kang SM, Seo B, Choi HY, Choi HS, Kwang S (2006) Anti-diabetic activity of SMK001, a polyherbal formula in streptozotocin induced diabetic rats: therapeutic study. Biol Pharm Bull 29:477-482
33. Nazief AM, Hassan PS, Khalifa HM, Sorkar MS, Kamel EL (2020) Lipid based gliclazide nanoparticles for treatment of diabetes: formulation, pharmacokinetics, pharmacodynamics and sub acute toxicity study. Int J Nanomed 15:1129-1148

34. Devarajan PV, Sonavane GS (2007) Preparation and in vitro/in vivo evaluation of gliclazide loaded Eudragit nanoparticles as a sustained release carrier. Drug Dev Ind Pharm 33:101-111

35. Kumar N, Soni S, Singh T, Kumar A, Ahmad FJ, Bhatnagar A, Mittal G (2015) Development and optimization of gastro-retentive controlled-release tablet of calcium-disodium edentate and it's in vivo gamma scintigraphic evaluation. AAPS Pharm Sci Tech 16:1270-1280

36. Upadhyay M, Adena SKR, Vardhan H, Yadav SK, Mishra B (2019) Locust bean gum and sodium alginate based interpenetrating polymeric network microbeads encapsulating Capecitabine: improved pharmacokinetics, cytotoxicity \& in vivo antitumor activity. Mater Sci Eng C 104:109958

37. Setia A, Goyal N, Kansal S (2013) Development and optimization of enteric coated mucoadhesive microspheres of duloxetine hydrochloride using 32 full factorial design. Int J Pharm Invest 3:141

38. Acharya S, Patra S, Pani NR (2014) Optimization of HPMC and carbopol concentrations in non-effervescent floating tablet through factorial design. Carbohydr Polym 102:360-368

39. Sahu BP, Das MK (2013) Optimization of felodipine nanosuspensions using full factorial design. Int J PharmTech Res 5:553-561

40. Wilding IR, Coupe AJ, Davis SS (2001) The role of $Y$-scintigraphy in oral drug delivery. Adv Drug Deliv Rev 46(103-124):24

41. Chowdary KP, Rao YS (2003) Design and in vitro and in vivo evaluation of mucoadhesive microcapsules of glipizide for oral controlled release: a technical note. AAPS PharmSciTech 4:2-7

42. Sharma A, Bhattacharya A (2010) Enhanced biomimetic sequestration of $\mathrm{CO}_{2}$ into $\mathrm{CaCO} 3$ using purified carbonic anhydrase from indigenous bacterial strains. J Mol Catal B Enzym 67:122-128

43. Gaudio PD, Colombo P, Colombo G, Russo P, Sonvico F (2005) Mechanisms of formation and disintegration of alginate beads obtained by prilling. Int J Pharm 302:1-9

44. Ostberg T, Lund EM, Graffner C (1994) Calcium alginate matrices for oral multiple unit administration: IV. Release characteristics in different media. Int J Pharm 112:241-248

45. Nayak AK, Hasnain MS, Beg S, Alam MI (2010) Mucoadhesive beads of gliclazide: design, development, and evaluation. Sci Asia 36:319-325

46. Tesfay D, Abrha S, Yilma Z, Woldu G, Molla F (2020) Preparation, optimization, and evaluation of epichlorohydrin cross-linked enset (Ensete ventricosum (Welw.) Cheeseman) starch as drug release sustaining excipient in microsphere formulation. Biomed Res Int 8:55-60

47. Dorniani D, Zobir M, Shaari AH, Ahmad Z (2013) Preparation and characterization of 6 Mercaptopurine-coated magnetite nanoparticles as a drug delivery system. Drug Des Dev Ther 7:1015-1026

48. Taha MO, Aiedeh KM, Al-Hiari Y, Al-Khatib H (2005) Synthesis of zinc-cross linked thiolated alginic acid beads and their in vitro evaluation as potential enteric delivery system with folic acid as model drug. Pharmazie 60:736-742

49. Ozoude CH, Azubuike CP, Ologunagba MO et al (2020) Formulation and development of metformin-loaded microspheres using Khaya senegalensis (Meliaceae) gum as co-polymer. Futur J Pharm Sci 6:120

50. Pal D, Nayak AK (2012) Novel tamarind seedpolysaccharide-alginate mucoadhesive microspheres for oral gliclazide delivery: in vitro-in vivoevaluation. Drug Delivery 19:123-131

51. Davis TME, Daly F, Walsh JP et al (2001) Pharmacokinetics and pharmacodynamics of gliclazide in Caucasians and Australian Aborigines with type 2 diabetes. Br J Clin Pharmacol 49:223-230

52. Davis TME, Daly F, Walsh JP et al (2006) Pharmacokinetics and pharmacodynamics of gliclazide in Caucasians and Australian Aborigines with type 2 diabetes. Br J Clin Pharmacol 49:223-230

53. Pal D, Nayak AK (2011) Development, optimization, and anti-diabetic activity of gliclazide-loaded alginate-methyl cellulose mucoadhesive microcapsules. AAPS PharmSciTech 12:1434-1441

54. Prajapati SK, Tripathi P, Ubaidulla U, Anand V (2008) Design and development of gliclazide mucoadhesive microcapsules: in vitro and in vivo evaluation. AAPS PharmSciTech 9:224-230

55. Hamed E, Sakr A (2001) Application of multiple response optimization technique to extended release formulations design. J Control Release 73:329-338 
56. Ye G, Wang S, Heng PWS, Chen L, Wang C (2007) Development and optimization of solid dispersion containing pellets of itraconazole prepared by high shear pelletization. Int J Pharm 337:80-87

57. Chowdary KPR, Rao YS (2003) Design and in vitro and in vivo evaluation of mucoadhesive microcapsules of glipizide for oral controlled release: a technical note. AAPS PharmSciTech 4:39

\section{Publisher's Note}

Springer Nature remains neutral with regard to jurisdictional claims in published maps and institutional affiliations.
Submit your manuscript to a SpringerOpen ${ }^{\circ}$ journal and benefit from:

- Convenient online submission

- Rigorous peer review

- Open access: articles freely available online

- High visibility within the field

- Retaining the copyright to your article

Submit your next manuscript at $\boldsymbol{\nabla}$ springeropen.com 